\title{
A Survey on the Mathematical Foundations of Axiomatic Entropy: Representability and Orderings
}

\author{
María-Jesús Campión ${ }^{1,+}+$ (D) , Cristina Gómez-Polo ${ }^{2,+}$ (D) , Esteban Induráin ${ }^{3, *,+}$ (i) \\ and Armajac Raventós-Pujol 1,+ (DD \\ 1 Institute for Advanced Research in Business and Economics (Inarbe), Departamento de Matemáticas, \\ Universidad Pública de Navarra, 31006 Pamplona, Spain; mjesus.campion@unavarra.es (M.-J.C.); \\ armajac.raventos@unavarra.es (A.R.-P.) \\ 2 Institute for Advanced Materials (InaMat), Departamento de Física, Universidad Pública de Navarra, \\ 31006 Pamplona, Spain; gpolo@unavarra.es \\ 3 Institute for Advanced Materials (InaMat), Departamento de Matemáticas, Universidad Pública de Navarra, \\ 31006 Pamplona, Spain \\ * Correspondence: steiner@unavarra.es; Tel.: +34-948-169-551; Fax: +34-948-166-057 \\ + These authors contributed equally to this work.
}

Received: 23 March 2018; Accepted: 3 May 2018; Published: 8 May 2018

\begin{abstract}
Different abstract versions of entropy, encountered in science, are interpreted in the light of numerical representations of several ordered structures, as total-preorders, interval-orders and semiorders. Intransitivities, other aspects of entropy as competitive systems, additivity, etc., are also viewed in terms of representability of algebraic structures endowed with some compatible ordering. A particular attention is paid to the problem of the construction of an entropy function or their mathematical equivalents. Multidisciplinary comparisons to other similar frameworks are also discussed, pointing out the mathematical foundations.
\end{abstract}

Keywords: ordered structures; numerical representations; entropy; representability; utility functions

\section{Introduction}

Entropy Theory can be compared to several other interdisciplinary mathematical theories that involve numerical representations of ordered structures (see, e.g., [1]). In particular, an appealing similarity arises between the utility representation problem in Economics and the search for entropy functions in Thermodynamics. This has been analyzed by paying attention to different aspects of the kind of representability that could be encountered (see [2-11]). Apart from Utility Theory in Economics, other theories, e.g., Measurement Theory in Psychology and Cognitive Sciences (see [12,13]), Shannon Entropy in Computation [14]) and Finance (see [15,16]), or Fuzzy Set Theory (see [17]), give rise to quite similar problems. Basically, we may say that all of them try to implement some ad hoc method or construct a suitable tool to convert qualitative scales into quantitative or numerical ones.

This is the clue.

Thus, in the case of Utility Theory in Economics, the device would be the concept of a numerical representation or utility function, whereas, in the case of Entropy Theory in Thermodynamics, the tool will be the notion of an entropy function. These theories share a common mathematical background.

Just to put an appealing example of a manuscript that analyzes the theoretical grounds of Entropy Theory in Thermodynamics, we choose an interesting paper by Cooper (see [18]). We have selected that paper by Cooper ([18]), dated 1967, because it shows a clear formulation to compare axiomatic Thermodynamics to representability theory of ordered structures in Mathematics. Indeed, it was published in the Journal of Mathematical Analysis and Applications, as an attempt to bring 
closer physical notions arising in Thermodynamics to pure mathematicians, by means of a rigorous mathematical setting. Needless to say, we are indeed aware that other (much more modern) axiomatic approaches to the concept of entropy have arisen in Physics. For instance, in an excellent survey by Lieb and Yngvason [19] (dated 1999), an axiomatic foundation of the second law of thermodynamics that includes the construction of entropy can be achieved in a more physical way. This approach is, in some sense, more general than Cooper's one in [18], in that it does not require the concept of empirical temperature. Nevertheless, and we insist in this key fact that, from the point of view of mathematicians, those physical approaches have exactly the same mathematical treatment, namely the use of representability theory of ordered structures to convert qualitative scales into quantitative ones. The new quantitative scales are defined through some entropy function in the physical setting. Thus, in the aim of building bridges, we have decided to follow Cooper's ideas, since, in our opinion, his presentation is perhaps more didactical, despite more modern approaches having appeared in Physics in the last fifty years.) In that key work, the author studies the foundations of Thermodynamics and the existence of entropy functions on state spaces of thermodynamic systems. Three different formulations of the second law of Thermodynamics from to Clausius, Kelvin and Carathéodory (see, e.g., [20,21]) are considered and it is proven that the satisfaction of Carathéodory's axioms (see, e.g., [22,23]) is not sufficient for the existence of an entropy function even in simple spaces. Cooper studies this problem by formulating a concept of an accessibility relation $\rightsquigarrow$ on the state space (also known as the phase system) $\mathcal{S}$ of a thermodynamical system. That relation on $\mathcal{S}$ is a total preorder, and an entropy function is defined to be a real-valued function on $\mathcal{S}$ that preserves $\rightsquigarrow$.

Even if we can mathematically justify the existence of an entropy function, a new problem appears, namely, the construction of a suitable one. To prove the existence and to describe a construction are horses of a different color. In this sense, it could be reasonable to compare how mathematicians and, say, physicists, argue even when the theories they are studying have the same mathematical roots. To simplify greatly, we may say that sometimes the physicist has at hand the result of an experiment or else the evidence of a real-life phenomenon, so that she/he wants to mathematically justify what happens and why. In this situation, the starting point is, so-to-say, that experiment or evidence, and Mathematics acts just as a theoretical support to conclude that everything is in good order. People who research in abstract pure mathematics, however, almost always start from an abstract system of axioms to build a theory, and nothing more. Everything should be obtained from the axioms, and even real-life evidence and situations should appear as "particular cases" of the corresponding theory that emanates from the axiomatic system involved. These two ways of thinking have, obviously, many points in common. However, they also have intrinsic differences (for a nice discussion about how mathematicians vs. physicists argue, see, e.g., [24]). A clear example of the above discussion appears when studying entropies. Most of the classical definitions of an entropy arising in Thermodynamics use some sort of path-integral. In Mathematics, however, if starting from a purely abstract point of view we deal with a totally ordered set satisfying some technical conditions that warrant the existence of a numerical representation (also known as order-isotony or utility function), the fact of that representation being done through a path-integral is quite difficult and unintuitive. In a mathematical abstract setting, only in very special cases the construction of a suitable integral that represents the ordered structure can be done in a small number of steps.

Therefore, it seems necessary here to make a discussion about the theoretical existence of a representation (e.g., an entropy) and its practical construction.

These problems, certainly related and complementary, are of a very different nature.

By the way, Boltzmann's works extended the understanding of thermodynamic entropy towards a statistical perspective. Gibbs (see [25]) brought the notion of grand canonical ensemble concept and introduced statistical entropy in classical continuous systems. Von Neumann then presented the so-called now Von Neumann entropy in quantum systems (see [26]). In addition, other than development in Physics, Claude Shannon (see [14]) independently introduced in 1948 an alternative axiomatic approach to justify the form of a concept of entropy related to communication problems in 
Information Theory. Shannon setting uses three axioms that are actually equivalent to those issued by Carathéodory and Cooper. In fact, E. T. Jaynes' pioneer works (see [27]) demonstrated that the forms of thermodynamic, statistical and Von Neumann entropy are actually rooted in the same mathematical bases, namely, they can be stated by using the same three axioms that Shannon used in [14], and this leads to equivalent forms of entropy, each of them for its corresponding context. Thus, despite having selected Cooper's approach focusing on axiomatic entropy in Thermodynamics, we could have also alternatively chosen Shannon's setting in Information Theory (as well as other mathematically equivalent ones) to start with. Furthermore, following Bayesian interpretation of probability theory, Laplace's insufficient principle and Gibbs's ensemble treatment, E. T. Jaynes argued that entropy is not only a physical quantity representing scale of order but also a tool for inductive inference. This topic has been investigated in the past decades by several scholars including J. Shore, R. Johnson, J. Skilling, C. Rodríguez, A. Caticha and K.H. Knuth (see, e.g., [28,29]), thus leading to a whole new understanding of the meaning of entropy, from interdisciplinary mathematical, physical and philosophical angles. In addition, there exist past related works on axiomatic entropy in the teleparallel alternative of general relativity with a non-minimal coupling between the gravity and scalar field (see, e.g., [30]). In some of these studies, ordering relations constitute a basis to obtain laws, as we do in the present paper.)

\subsection{Contents of the Paper}

The present paper constitutes a survey on the main mathematical theories and results that support the concept of an entropy arising in Physics and Chemistry among other neighboring disciplines. Then, a critical discussion is made about how mathematicians have achieved some of the key results, and what could be the scope of such results in entropy theory. A particular attention is paid to the construction of entropy functions. Other possible miscellaneous applications and similar theories are also commented as a by-product.

The schema of the paper goes as follows:

After the Introduction, in Section 2, we select the key definitions, both on entropies and ordered structures, that are necessary to start with. In Section 3, we pay attention to the representability of total preorders, as well as to the construction of utility functions (also known as order-isotonies). In Section 4, we analyze the concepts of mixing and competition, commonly encountered in various entropy settings, in the light of numerical representations of certain ordered structures, that may be defined on a set endowed with some algebraic operation compatible with the ordering, and perhaps some related topology, too. Sometimes, these ideas of looking for entropy functions that preserve some algebraic operation and/or are continuous with respect to some topology, appear directly in a set of axioms, as in [18] or much more recently in [19]. However, from the mathematical point of view, the representability theory of algebraic and/or topological structures has special features, and should be analyzed after a well understanding of the representability of, say, total preorders (without any additional structure). To avoid misunderstanding, we warn the reader that, here, the word "topology" or the expression "topological structure" should be understood only in the sense of General Topology (see [31]) and not in the sense of a "topological configuration of discrete structures" that is typical, for instance, in the study of graphs and networks, where concepts related to entropy, as the so-called "graph entropy", are also of common use (see, e.g., [32]).Other expressions, such as "topological sorting" (see, e.g., [33,34]) used in graph theory to obtain a suitable linear order on the nodes of a directed acyclic graph, are also disparate from the concept of "topology" throughout the present manuscript. Basically, we work with a topology as the collection of open subsets of a given set. We do this, mainly, to handle continuity of functions.) This is the reason that forces us to study this question in a separate section. Then, Section 5 is devoted to studying the representability numerical of interval orders and semiorders. Section 6 analyzes questions relative to intransitivities related to physical or chemical processes. This is made in the light of suitable ordered structures, namely interval orders and semiorders. As far as we know, the use of this kind of orderings in entropy theory in Thermodynamics or Chemistry is new. Interval orders are used to interpret intransitivities 
(see [1]), whereas semiorders, that constitute a special case of interval orders, can be used to interpret situations in which two systems cannot be distinguished until they differ in a fixed amount, called threshold or "quantum". Therefore, the novelty of the use of semiorders is that they constitute a helpful mathematical tool to deal with quantum theories related to entropy. In Section 7, we pay special attention to the construction of numerical representations.

This study is necessary, in our opinion, because the construction of numerical representations of ordered structures in a pure mathematical abstract setting could seriously differ from the typical understanding of an entropy in Thermodynamics as, say, a physical concept given by a suitable path-independent integral.

Thus, we should explain how representations of orderings can be built in a way so that they are defined by some sort of integral.

The manuscript is closed with a Discussion (Section 8) and a final Section 9 of conclusions, open problems and further comments, pointing out several lines of research to complete the panorama.

\subsection{Objectives, Methodology and Original Work}

We warn the reader that this paper, despite presenting interdisciplinary results, has been conceived as a mathematical one. We think that analyzing the mathematics that appears in the basis of axiomatic theories on entropy could help other people working in several disciplines, perhaps disparate.

It is not our intention to compare one another all the axiomatics that have been introduced in Physics to handle the concept of entropy in quite different settings, mainly in Thermodynamics. Instead, it is our aim to show how the theory of representability of ordered structures is the mathematical cornerstone on which the most typical axiomatics of entropy lean. That is, instead of working at some of the various physical levels or in some concrete physical theory, we go to the background level of pure abstract mathematics.

To do so, we have chosen some particular axiomatics of entropy arising in Physics or Information Theory, namely the one due to Carathéodory issued in 1909 and then improved by many authors such as Cooper, Lieb and Yngvason, or even Shannon, among others. As commented before, we do not think it is effective to compare and analyze each one of the possible axiomatics.

When observing most of these existing systems of axioms, we notice that most of them set a list of postulates that basically establish that the state space is endowed with a binary relation, usually called accessibility, that is a linear order or a total preorder. Then, they look for a real-valued function called entropy that preserves the ordering and is a quantitative scale to compare states. This is done from the axioms, by proving the corresponding existence theorem.

Therefore, most of these axiomatics share the same mathematical background. Thus, it seems crucial to directly analyze that abstract basis, since understanding it well, we could use the subsequent abstract mathematical results that derive from it, namely the fundamentals of the theory of the numerical representability of ordered structures, to support each of those physical theories at least in their axiomatical setting.

Of course, this last step leads to some changes of nomenclature, depending on the concrete theories involved. However, and we insist on this, the mathematical basis is the same.

Bearing this in mind, our objective here is to analyze the order theoretical questions that arise in those axiomatics of entropy. Thus, we develop a rigorous mathematical battery of results that could help us the particular features of each possible interdisciplinary situation.

In those axiomatics we also observe that, from the abstract mathematical point of view, some structures appear there amalgamated. That is, some axioms are of ordinal stuff, some other ones deal with algebraic operations and several others involve continuity, that is a topological concept. All of them appear mixed or combined. Unfortunately, from the point of view of the mathematical theory of numerical representability of ordered structures, this could be confusing and sometimes misleading. Thus, a formal theory should begin by studying the representability of linear orders and total preorders, with no extra structure. Then, new compatible structures can be added as algebraic 
operations first, and then continuity with respect to some topology. In general, it is much clearer to study these mathematical additional structures separately.

Once the main mathematical results have been obtained, one can come back to the axiomatics of entropy to rewrite or rearrange, if necessary, the set of axioms. Sometimes, some condition should be added ab initio to the state systems because, at least from the mathematical abstract setting, it cannot be taken for granted. For example, if we want to work with connected spaces, or if we want to deal with systems that can interact, we should postulate that in the axioms. In other words, an abstract set is neither given a priori a topology (to deal with connectedness or continuity), nor a sort of algebraic operation (to handle interactions or reactions).

The original work made in this paper can be summarized as follows:

1. Identify the mathematical background that most axiomatics of entropy share. That basis is the theory of numerical representability of linear orders and total preorders. It is also the basis of several other theories, for instance, Utility Theory in Economics.

2. Isolate the mathematical components of the axiomatics. In several axiomatics encountered in the literature, the consideration of several mathematical aspects of the phase systems appears as a mixture or list of properties. It is more convenient to analyze each aspect independently, since this also gives us a further idea of the scope of each property considered. Thus, it is mathematically reasonable to study first ordered structures without any additional condition, and then pass to consider algebraic ordered structures and finally continuity as well as other topological features.

3. Point out that nothing can be taken for granted a priori, if it has not been said in the corresponding axioms, and correct some mistakes arising in classical papers. Any property that is implicitly supposed but not declared could lead to mathematical mistakes as well as flaws in the proofs. This has already happened in $[18,19]$, among other sources. Perhaps due to implicitly assuming some properties that have not been declared, some classical results have flaws in the proofs or are based in intuitions that are false in the abstract setting. This happens in [18,35-37] (see also [2,3]). Apart from this, other mistakes of different nature arising, e.g., in [18], have also been corrected.

4. Suggest new axioms that should be added. This is necessary to obtain correct results and avoid the mistakes that are due either to false intuitions or to the fact of taking some properties for granted.

5. Identify the theory of numerical representability of interval orders as the key to deal with intransitivities. This fact is well known in the corresponding mathematical literature. However, as far as we know, it is uncommon in the analysis of axiomatics of entropy. Although several recent papers (see, e.g., [38]) have analyzed in depth intransitivities encountered in various physical or chemical processes, no use of the concept of an interval order as the main tool is observed there, as already commented in [1].

6. Show some parallelism among axiomatics of entropy and other interdisciplinary theories. In particular, we point out that several crucial results in the theory of numerical representability of ordered structures were indeed launched in journals devoted to the study of utility theory in Economics.

7. Use semiorders as the key mathematical concept to deal with intransitivities that are due to the existence of constant thresholds of discrimination. As in the case of interval orders, this seems to be new in the axiomatics of entropy. In the case of quantum theories, it could be crucial in future research to interpreting quanta as a thresholds of comparison, and work in the framework of the numerical representability of semiorders in the sense of Scott and Suppes (see $[39,40])$.

8. Analyze the construction of numerical representations of orderings, in particular when they are given by integrals. To achieve a theoretical result that states that a numerical representation must exist, because otherwise we arrive to a mathematical contradiction, is quite different from furnishing a construction of a suitable representation. We pay particular attention to the case in which the numerical representations are defined through some sort of integral. 


\section{Preliminaries}

\subsection{Ordered Structures}

From now on, $X$ denotes a nonempty set.

Definition 1. A binary relation $\mathcal{R}$ on $X$ is a subset of the Cartesian product $X \times X$. Given two elements $x, y \in X$, we use the standard notation $x \mathcal{R} y$ to express that the pair $(x, y)$ belongs to $\mathcal{R}$.

Associated to a binary relation $\mathcal{R}$ on a set $X$, we consider its negation (respectively, its transpose) as the binary relation $\mathcal{R}^{c}$ (respectively, $\mathcal{R}^{t}$ ) on $X$ given by $(x, y) \in \mathcal{R}^{c} \Leftrightarrow(x, y) \notin \mathcal{R}$ for every $x, y \in X$ (respectively, given by $(x, y) \in \mathcal{R}^{t} \Leftrightarrow(y, x) \in \mathcal{R}$, for every $\left.x, y \in X\right)$. We also define the adjoint $\mathcal{R}^{a}$ of the given relation $\mathcal{R}$, as $\mathcal{R}^{a}=\left(\mathcal{R}^{t}\right)^{c}$.

Remark 1. The symbol $\neg$ is also commonly used to mean "negation", so that $\neg(x \mathcal{R} y)$ is interpreted as " $x \mathcal{R} y$ never occurs".

A binary relation $\mathcal{R}$ defined on a set $X$ is said to be:

(i) reflexive if $x \mathcal{R} x$ holds for every $x \in X$;

(ii) irreflexive if $\neg(x \mathcal{R} x)$ holds for every $x \in X$;

(iii) symmetric if $\mathcal{R}$ and $\mathcal{R}^{t}$ coincide;

(iv) antisymmetric if $\mathcal{R} \cap \mathcal{R}^{t} \subseteq \Delta=\{(x, x): x \in X\}$;

(v) asymmetric if $\mathcal{R} \cap \mathcal{R}^{t}=\varnothing$;

(vi) total if $\mathcal{R} \cup \mathcal{R}^{t}=X \times X$; and

(vii) transitive if $x \mathcal{R} y \wedge y \mathcal{R} z \Rightarrow x \mathcal{R} z$ for every $x, y, z \in X$.

In the particular case of a nonempty set where some kind of ordering has been defined, the standard notation is different. We include it here for the sake of completeness, since we use it throughout the present manuscript.

Definition 2. A preorder $\precsim$ on $X$ is a binary relation on $X$ which is reflexive and transitive. An antisymmetric preorder is said to be an order. A total preorder $\precsim$ on a set $X$ is a preorder such that if $x, y \in X$ then $(x \precsim y) \vee(y \precsim x)$ holds. A total order (i.e., an antisymmetric total preorder) is usually called a linear order.

If $\precsim$ is a preorder on $X$, then as usual we denote the associated asymmetric relation by $\prec$ and the associated equivalence relation by $\sim$ and these are defined by $x \prec y \Leftrightarrow(x \precsim y) \wedge \neg(y \precsim x)$ and $x \sim y \Leftrightarrow(x \precsim$ $y) \wedge(y \precsim x)$.

Remark 2. In some contexts arising in Economics, a total preorder is called a preference.

Definition 3. An interval order $\prec$ is an asymmetric binary relation on $X$ such that $(x \prec y) \wedge(z \prec t) \Rightarrow$ $(x \prec t) \vee(z \prec y)(x, y, z, t \in X)$. Its corresponding adjoint is denoted $\precsim$, so that $a \precsim b \Leftrightarrow \neg(b \prec a)$. This relation $\precsim$ is called the weak preference associated to $\prec$. By the way, $\prec$ is also called a strict preference defined on $X$. In addition, the binary relation $\sim$ defined by $a \sim b \Leftrightarrow(a \precsim b) \wedge(b \precsim a)$ is said to be the indifference associated to $\prec$. An interval order $\prec$ is said to be a semiorder if, for every $a, b, c, d \in X$ it holds true that $a \prec b \prec c$ implies that $a \prec d$ or $d \prec c$ holds true.

Remark 3. The intuition behind the definition of an interval order, is that the elements $x, y, z, t$ that appear in the condition, also known as the Ferrers property, given by $(x \prec y) \wedge(z \prec t) \Rightarrow(x \prec t) \vee(z \prec y)$ behave as if they were the end-points of two intervals of the real line $\mathbb{R}$. Indeed, given two intervals $[x, y]$ and $[z, t]$ of the real line, with respect to the usual order $\leq$ in $\mathbb{R}$ it is clear that the condition $(x<y) \wedge(z<t) \Rightarrow(x<t) \vee(z<y)$ holds true. This justifies the nomenclature interval order for this kind of orderings on an abstract nonempty set $X$. In addition, the intuition behind the concept of a semiorder could be the following one: suppose that $a, b, c, d$ are points in a line and, given $x, y \in X$, we interpret $x \prec y$ as "at least an inch separes $x$ from $y$ ". Notice that 
"an inch" acts here as, so-to-say, a threshold of discrimination. The nomenclature "semiorder" is attributed to R. Duncan Luce, who introduced it in [39]. However, the concepts of interval order and semiorder had already been considered in the literature much earlier (see, e.g., [41]), under different names.

Remark 4. It is well-known that given an interval order $\prec$ on a set $X$, the associated relations $\precsim$ and $\sim$ may fail to be transitive (see, e.g., [39,42,43]). However, the strict preference $\prec$ is always transitive. Moreover, a property usually known as pseudo transitivity namely $s \prec t \precsim x \prec u \Rightarrow s \prec u$ for every $s, t, x, u \in X$ also holds true (see, e.g., [42-46] for further details).

\subsection{Entropy}

Even for physicists, the concept of entropy is complex and difficult to grasp. It has been given many definitions, interpretations, explanations and applications. The corresponding literature is vast and somewhat confusing. It is a key concept in Thermodynamics. Indeed, it is commonly accepted that Thermodynamics was created during the nineteenth century by engineers interested in the development of heat machines (see, e.g., [47]). Its main pioneers were Sadi Carnot, Rudolph Clausius, Benoît Claperyon, James Clerk Maxwell, and William Thomson (Lord Kelvin). Thermodynamics is based on two laws: the first one states that the energy is conserved, whereas the second one says that not all transformations are possible. The second law invokes a quantity called the entropy, whose evolution in time measures the evolution of a system.

Let us comment in brief some classical definitions and/or interpretations of this crucial concept, namely entropy:

It was Rudolf Clausius who, in 1865, explicitly launched the notion of entropy (see [48]) as $S=\frac{Q}{T}$ where $S$ denotes the entropy, $Q$ is the heat content (also known as internal heat energy) of a given system and $T$ stands for its temperature. Here, it is usually understood that a system can only have a temperature if it is in thermodynamical equilibrium. Entropy is only defined for this kind of systems in equilibrium. Thus, the temperature must be taken as being a constant. Therefore, we arrive at $\Delta S=\frac{\Delta Q}{T}$, where the symbol " $\Delta$ " stands for a finite increment. Thus, $\Delta S$ indicates a change in entropy as, say, $\Delta S=S_{1}-S_{2}$, where $S_{1}$ and $S_{2}$ denote the entropies of two different equilibrium states. (This setting is still common nowadays in several contexts arising in Physics, see, e.g., [49].)

By the way, Clausius and especially Carnot, among other physicists, were interested in how to convert mechanical work into heat energy, and vice versa. This idea can lead us to an alternate form of the previous equation $\Delta S=\frac{\Delta Q}{T}$. If we pump energy $\Delta U$ into a system, a part of the energy (but not all of it) goes into the internal heat content, $Q$, making $\Delta Q$ a positive quantity. The remaining energy could be understood as the mechanical work done by the system $(\Delta W$, such as a hot gas pushing against a piston in a car engine). Thus, $\Delta Q=\Delta U-\Delta W$, where $\Delta U$ is the energy input to the system, and $\Delta W$ is the part of that energy that goes into doing work. The difference between them is the amount of energy that does not participate in the work, and goes into the heat reservoir as $\Delta Q$. Thus, a simple substitution leads to rewrite the previous equation as $\Delta S=(\Delta U-\Delta W) / T$.

From a mathematical point of view, when increments or change of heat and entropy can be as small as we please, it is also common to use differential Calculus and write the corresponding formula as, say, $d S=\frac{d Q}{T}$. In fact, in classical Thermodynamics, when considering a closed homogeneous system in which there is only room for reversible processes, it happens that $\oint \frac{d Q}{T}=0$. That means that the integral $\int_{L} \frac{d Q}{T}$ is path-independent. This gives rise again to defining a state function, $S$, called entropy, that accomplishes that: $d S=\frac{d Q}{T}$.

Following Cooper (see [18]), we may point out that a nub result of classical macroscopic thermodynamics is the deduction of the existence and properties of entropy from several forms of the second law. These forms are actually derived from experience, practice and suitable experiments. The arguments involve, in addition to the first and second laws of thermodynamics, assumptions about heat and temperature, as well as on the properties of perfect gases. Thus, in the later 1800s, Maxwell, Ludwig Boltzmann and Josiah Willard Gibbs extended the ideas of classical thermodynamics, 
through the new "molecular theory" of gases, into the domain we now call statistical mechanics. In classical thermodynamics, we deal with single extensive systems, whereas, in statistical mechanics, we recognize the role of the tiny constituents of the system. The temperature, for instance, of a system defines a macrostate, whereas the kinetic energy of each molecule in the system defines a microstate. The macrostate variable, temperature, is recognized as an expression of the average of the microstate variables, an average kinetic energy for the system. Hence, if the molecules of a gas move faster, they have more kinetic energy, and the temperature naturally goes up.

However, the deduction of properties of entropy in that way was criticized by some mathematical physicists, e.g., Max Born (see [50]). These criticisms lean on the fact that these assumptions involve redundancies.

It was Constantin Carathéodory who, in 1909, introduced an elegant development that is immune to those criticisms because it does not involve heat and temperature as initial concepts.

Classical formulations of the second law of thermodynamics are as follows (see, e.g., the survey [19] for a further account):

(1) (Clausius) No process is possible in nature, the sole results of which are that heat is transferred from a colder body to a hotter one.

(2) (Kelvin) No process is possible, the sole result of which is that a body is cooled and work is done. This characterizes the impossibility of a "perpetuum mobile".

(3) (Planck) No process is possible, the sole result of which is that a body is cooled and a weight raised.

(4) (Carathéodory) In any neighborhood of any state $s$ of an isolated thermodynamic system, there exist states which cannot be reached from $s$ by any possible processes.

Carathéodory (see [51]) and then several authors, such as Cooper (see [18]), or Lieb and Yngvason (see [19]), among many others, investigated how far the existence of an entropy function is a consequence of relations of inaccessibility between states. To do so, they studied the relationship between those different statements of the second law of thermodynamics, as well as a system of axioms, formerly introduced by Carathéodory (see $[3,4,18,22,51]$ ) to deal with the concept of accessibility. From these axioms and other technical assumptions (as discussed in [2-4]), the existence of an entropy function would follow. In these axioms, the state space $S$ of a thermodynamical system is assumed to have been endowed with a relationship $\rightsquigarrow$ called accessibility, such that a notation as $s \rightsquigarrow t$ can be interpreted as "a transition from the state $s$ to the state $t$ is possible". An entropy function is then defined as a map $f$ from the state space $S$ into the real line such that $s \rightsquigarrow t$ holds if and only if $f(s) \leq f(t)$. One of the Carathéodory axioms says that $\rightsquigarrow$ is a total preorder, namely:

(i) For any two states $s$ and $t$, either $s \rightsquigarrow t$ or $t \rightsquigarrow s$ hold true.

(ii) For any three states $r, s, t$, if $r \rightsquigarrow s$ and $s \rightsquigarrow t$, then $r \rightsquigarrow t$ holds.

(iii) For any state $s, s \rightsquigarrow s$ holds true.

Remark 5. Notice that the asymmetric part of the accessibility relation $\rightsquigarrow$ would constitute a mathematical tool to study situations arising in Physics, Chemistry, Engineering, etc., where an irreversible process arises (see, e.g., [52]). If we denote by $\rightsquigarrow *$ the asymmetric part of $\rightsquigarrow$, a notation as $s \rightsquigarrow^{*} t$ would be interpreted as "a transition from the state $s$ to the state $t$ is possible, whereas a transition from $t$ to $s$ can never occur." In other words, we interpret that "if a transition from s to $t$ is made, the process is irreversible: there is no way back from t to s".

The other axioms are of topological nature, involving a sort of continuity of the accessibility relation with respect to some suitable topology for which the state space is separable. With these axioms, it is proven that there exists a continuous entropy function for the binary relation $\rightsquigarrow$ of accessibility (see, e.g., Theorem 1 in [18]). A more or less similar situation arises in the more recent survey [19], where axioms of algebraic nature are mixed with the ones that just lead to the existence of a numerical representation (entropy, in this setting) of a totally preordered set. 
Remark 6. Unfortunately, as discussed in depth in [1-4], Cooper's proof of his Theorem 1 in [18] is incorrect unless some other conditions are asked a priori to be accomplished for the state space S. One of these possible conditions is the connectedness, already assuming separability, of the state space (see [53]). Other sufficient condition is second countability (see [35,54]). Quite probably, both Carathéodory and Cooper had some of those extra conditions implicitly in mind. For instance, connectedness is quite natural, in the spirit of the renowned Newton's sentence "Natura non facit saltum". In Cooper's words (see [18]):

$<<$ The physical model for a thermodynamic system is a system isolated from the external world by barriers impassible to heat but through which mechanical, electromagnetic, gravitational or other interactions with the external world are possible: these interactions are summed up under the term interactions of the ground theories. The ground theories are the parts of physics established independently of thermodynamics such as mechanics and electromagnetic theory. Within the thermodynamic system, subsystems capable of being isolated by barriers impassible to heat may exist, but it must be assumed that these internal barriers can be removed. $>>$

From a topological point of view, Cooper's words could be interpreted in the sense that the "barriers" there mentioned would provoke a disconnection of the state space. In other words, when the barriers are removed, the space becomes connected as regards its topology.

As a part of the study of representability of ordered structures, in Section 3, we review again Cooper's approach to the Carathéodory setting, pointing out how the flaws or gaps in some proofs can be mended.

\section{Numerical Representability of Total Preorders}

\subsection{Representable Total Preorders}

Let $X$ be a nonempty set, and $\mathcal{R}$ a binary relation on $X$. Depending on its properties, we could try to interpret $\mathcal{R}$ as a qualitative scale on $X$. For instance, given $x, y \in X$, if $x \mathcal{R} y$ we could think that "the element $y$ is as least as good as the element $x$ ". Obviously, we would like then to convert that qualitative scale into a numerical or quantitative one, since it is easier and more natural to compare numbers rather than elements of an abstract given set. Therefore, we would like to have at hand a function $f: X \rightarrow \mathbb{R}$ such that, given $x, y \in X, x \mathcal{R} y$ holds if and only if $f(x) \leq f(y)$. However, by the properties of the relationship " $\leq$ " among real numbers, the mere existence of a function $f$ that satisfies the aforementioned condition immediately carries very strong restrictions on the binary relation $\mathcal{R}$. Indeed, it is straightforward to see that, when a such function $f$ exists, the binary relation $\mathcal{R}$ must a fortiori be a total preorder.

Remark 7. The converse fact is not true, as we analyze below in Section 3. That is, not every total preorder $\precsim$ on $X$ gives rise to the existence of a real-valued function $f$ such that, given $x, y \in X, x \mathcal{R} y \Leftrightarrow f(x) \leq f(y)$. This inspires the following definition.

Definition 4. Let $\precsim$ stand for a total preorder defined on the nonempty set $X$. The total preorder $\precsim$ is said to be representable if there exits a real-valued function $u: X \rightarrow \mathbb{R}$ such that for any $x, y \in X$ it holds true that $x \precsim y \Leftrightarrow u(x) \leq u(y)$. The function $u$ is usually called "utility function" (also known as order-isotony or numerical representation) for the given preorder $\precsim$ on $X$.

Given a total preorder $\precsim$ defined on $X$, we also consider its associated equivalence relation $\sim$, also known as the indifference relation with respect to $\precsim$. If we collapse each equivalence class to a point, we immediately define a binary relation $\preceq$ on the quotient set $X / \sim$ of indifference classes, by declaring that, for every $x, y \in X,[x] \preceq[y] \Leftrightarrow x \precsim y$. Here, $[x]$ (respectively, $[y]$ ) stands for the indifference class of the element $x$ (respectively, $y$ ), namely $[x]=\{z \in X: x \sim z\}$. Since $\precsim$ is a total preorder, the binary relation $\preceq$ is actually a linear order on $X / \sim$. In addition, it is clear that the given total preorder $\precsim$ is representable if and only if its associated linear order $\preceq$ is. In other words, if we do not ask for extra conditions of algebraic or topological nature (e.g., some sort of continuity), the 
problem of the numerical representability of total preorders is equivalent to that of the numerical representability of linear orders. This fact immediately generates examples of non-representable total preorders. If we accept the Zermelo-Fraenkel axioms of Set Theory (see, e.g., [55]) or at least the well ordering principle (also known as Zermelo's theorem), we may consider a set $X$ whose cardinality is strictly bigger than that of the real line $\mathbb{R}$ (here the power set $2^{\mathbb{R}}$ suffices) equipped with a well ordering. Obviously, there is no room in $\mathbb{R}$ to represent that well ordering, since we would need to have a codomain much larger than $\mathbb{R}$. (Notice that the codomain should have at least the cardinality of $2^{\mathbb{R}}$.) Having this fact in mind, we may wonder why several authors, in different contexts, claim that numerical representations always exists. Thus, in a context of Utility Theory in Economics, Hicks (see p. 19 in [36]) claimed that "if a set of items is strongly ordered, it is such that each item has a place of its own in the order; it could, in principle, be given a number". In the same way, one may think that the aforementioned accessibility axiom in Carathéodory and Copper's approach to entropy is already enough to guarantee the existence of an entropy function. However, this is false: other additional conditions are required ab initio. Perhaps these common beliefs hang from false intuitions in which we had always in mind spaces that behave as the real line $\mathbb{R}$, or maybe as a real Euclidean space $\mathbb{R}^{n}$. However, these intuitions can never be taken as properties shared by any possible abstract set we may deal with.

In conclusion: we need to have at hand characterizations of the numerical representability of total preorders, valid for the general case, that is, for any totally ordered nonempty abstract set. To do so, we introduce now some necessary definitions.

Definition 5. Let $\precsim$ be a total preorder defined on a nonempty set X. The preorder $\precsim$ is said to be separable in the sense of Debreu (see $[35,37,44,54]$ if there exists a countable subset $D \subseteq X$ such that given $x, y \in X$ with $x \prec y$, there exists some $d \in D$ satisfying that $x \precsim d \precsim y$. It is said to be perfectly separable (see, e.g., [56]) if there exists a countable subset $D \subseteq X$ such that given $x, y \in X$ with $x \prec y$, there exists some $d \in D$ satisfying that $x \prec d \precsim y$.

Remark 8. For standard definitions of basic concepts arising in General Topology we refer to [31].

Definition 6. Let $\precsim$ be a total preorder defined on a nonempty set X. Consider on $X$ the topology $\tau_{\precsim}$ a subbasis of which is $\{\varnothing\} \cup\{X\} \cup_{x \in X}\{z: z \prec x\} \cup_{t \in X}\{y: t \prec y\}$. This topology is said to be the order topology on $X$ relative to $\precsim$.

If the set $X$ is already endowed with a topology $\tau$, we say that the preorder $\precsim$ is continuous with respect to $\tau$, if the order topology $\tau_{\precsim}$ is coarser than $\tau$ (i.e., $\tau$ is finer than $\tau_{\precsim}$ ). In other words, $\precsim$ is continuous with respect to $\tau$ whenever the so-called contour sets $\{y \in X: y \prec x\}$ and $\{z \in X: x \prec z\}$ are open in the topology $\tau$, for every $x \in X$. Dually, if $\precsim$ is continuous with respect to $\tau$, it is also said that $\tau$ is a natural topology for $\precsim$.

Theorem 1. Let $\precsim$ be a total preorder defined on a nonempty set X. The following statements are equivalent:

(i) $\precsim$ is separable in the sense of Debreu.

(ii) $\precsim$ is perfectly separable.

(iii) There exists a countable subset $D \subseteq X$ such that given $x, y \in X$ with $x \prec y$, there exists some $d \in D$ satisfying that $x \precsim d \prec y$.

(iv) There exists a countable subset $D \subseteq X$ such that, given $x, y \in X$ with $x \prec y$, there exists some $d_{1}, d_{2} \in D$ satisfying that $x \precsim d_{1} \prec d_{2} \precsim y$.

(v) The order topology relative to $\precsim$ satisfies the second countability axiom.

(vi) The order topology relative to $\precsim$ is metrizable and separable.

Proof. See, e.g., the first three chapters of [44] or Section 3 in [56].

Many other equivalent conditions that characterize the representability of a total preorder are encountered in the literature. 
Remark 9. The property introduced in Part (iv) of the statement of Theorem 1 is called separability in the sense of Jaffray, or separability in the sense of Herden (see $[57,58])$.

We may ask ourselves if the existence of a non-representable total preorder $\precsim$ on a nonempty set $X$, forces the cardinality of $X$ to be bigger than that of the real line, as in the discussion previous to Definition 5. The answer is negative, that is, there are nonempty sets of the same cardinality as $\mathbb{R}$ that admit a non-representable total preorder. A typical example of this kind is the lexicographic plane, defined as follows: Let the real plane $\mathbb{R}^{2}$ be endowed with the total order $\preceq_{L}$ given by $(x, y) \preceq_{L}(z, t)$ if and only if $x<z$ or $x=z, y \leq t \quad(x, y, z, t \in \mathbb{R})$. Intuitively, we may notice that, if there were a utility function representing $\precsim_{L}$ each vertical line should be mapped into an open interval of the real line $\mathbb{R}$. However, since in the real line any family of pairwise disjoint open intervals is countable, we would not have room enough to represent the whole plane $\mathbb{R}^{2}$, because the set of real lines is uncountable. (For further details, see [37,44,59,60], as well as p. 73 in [61], among many other possible sources.)

Remark 10. Coming back to results related to entropy, we point out that one of the main results in Cooper's paper ([18]), in the spirit of Carathéodory axioms, states that:

$<<$ Suppose that the state space $S$ is given a topology $\tau$ such that the topological space $(S, \tau)$ is topologically separable. Assume, in addition, that $S$ is equipped with an accessibility relation $\rightsquigarrow$ which is a total preorder. Suppose also that $\rightsquigarrow$ is continuous as regards $\tau$. Then, there exists a real-valued, continuous, entropy function for the accessibility relation $\rightsquigarrow$ defined on $S .>>$

However, unfortunately, this claim is not true, as proven, e.g., in [3]. An example to see this is a suitable subset of the lexicographic plane, namely the subset $[0,1] \times\{0,1\} \subsetneq \mathbb{R}^{2}$, endowed with the lexicographic order $\preceq_{L}$ and the corresponding order topology. It can be seen that such topological space is actually separable, however it does not satisfy the second countability axiom, as required by Theorem 1. The statement of Cooper's main result can be corrected by putting "satisfies the second countability axiom" instead of "is topologically separable". (Notice also that the satisfaction of the second countability axiom is an hereditary property, unlike topological separability, which is not hereditary in general). Another possibility is to say that $(S, \tau)$ is metrizable and separable.

Perhaps because some (false) intuitions were assumed a priori, but not explicitly declared, not only it was accepted or taken for granted that an entropy function would exist in typical processes arising in Physics or Chemistry, but also in other different contexts that share the same mathematical background (e.g., Utility Theory in Economics) it was also implicitly accepted that numerical representations would also exist. Obviously, Theorem 1 and the subsequent examples and comments show that this is not true. However, there was some reticences, at least among economists, to accept what Theorem 1 is saying, in the sense that they believed that "normal preferences will always admit a utility representation" (see, e.g., the aforementioned sentence due to Hicks [36]). Needless to say, to this respect we should immediately ask: What do you mean by a normal preference? At this respect, we quote here a famous anecdote due to G. Debreu, Nobel Prize in Economics in 1983:

$<<$ Debreu was asked about a "normal" or real-life example of a preference that does not admit a utility representation. He answered to his interlocutor as follows: -OK. Suppose that I will invite you to an excellent meal in a good restaurant. I know you well, and I am aware that you always prefer to eat as much as possible, and for sure you prefer to eat to drink. In addition, if I offer you some food accompanied by some wine you will prefer that to have the same amount of food but no wine. Well, if I denote by $f$ (respectively, by w) the amount of food (respectively, wine) in your meal, here your preferences are such that $(f, w)$ is better than $\left(f^{\prime}, w^{\prime}\right)$ provided that $f>f^{\prime}$ or else $f=f^{\prime}$ but $w>w^{\prime}$. I can straightforwardly prove that those preferences fail to be representable. $>>$

Once this anecdote was commonly known by economists, and also because the preferences in that Debreu's anecdote are lexicographic, the result or "theorem" that states that the lexicographic plane is not representable is, nowadays, popularly known as "the Glutony theorem". 


\subsection{Continuous Representability of Total Preorders}

When dealing with Entropy, it is typical to consider some topological assumptions, as Cooper did in [18]. In some other contexts arising in other disciplines, e.g., Utility Theory in Economics, it is also usual to have at hand some topology involved. For instance, when handling preferences, it is common to see how they act on points that are similar in a sense, or "close to" one another (where, to fix ideas, this "closeness" could have been established by a metric, a distance, or just a topology that tells us what do we mean by "neighborhood" of a point). That is, in several contexts involving total preorders, it is common to encounter some topological features. Of course, this immediately carries the necessity of studying properties as continuity of the numerical representations, if any, of those total preorders (e.g., preferences, accessibility relations, etc.) involved.

Evidently, if we intend to deal with properties related to continuous representability of a total preorder $\precsim$ defined on a nonempty set $X$ which is endowed with a topology $\tau$, it is natural to ask the preorder to be related in some way with the given topology.

The typical assumption is the continuity of $\precsim$ with respect to the topology $\tau$. (In other words, $\tau$ is a natural topology for $\precsim$ ). Notice that if a utility function $u: X \rightarrow \mathbb{R}$ is continuous with respect to the order topology $\tau_{\precsim}$ on $X$ and the usual Euclidean topology on the real line, it is also continuous with respect to any natural topology $\tau$ on $X$, and the usual one on $\mathbb{R}$.

Example 1. Consider the function $f: \mathbb{R} \rightarrow \mathbb{R}$ given by $f(x)=5 x$ if $x<0, f(x)=5 x+1$ if $0 \leq x<3$, $f(x)=5 x+3$ if $3 \leq x<7$ and $f(x)=5 x+10$ if $x \geq 7$. It is clear that such function $f$ is strictly increasing. Hence, it satisfies that $x \leq y \Leftrightarrow f(x) \leq f(y)$ holds true for every $x, y \in \mathbb{R}$. Thus, $f$ acts as a numerical representation (or utility function) for the usual order $\leq$ on the real line. Notice that in this case, through the function $f$ we represent $\leq$ also on the same real line endowed with the same linear order $\leq$. With respect to the usual topology on $\mathbb{R}$, the function $f$ has jump discontinuities at the points $x=0, x=3$ and $x=7$. By the way, the usual Euclidean topology on the real line is just the order topology $\tau_{\leq}$associated to the usual linear order $\leq$. Now, consider the following modification of $f$, namely the function $g: \mathbb{R} \rightarrow \mathbb{R}$ given by $g(t)=5 t$, for all $t \in \mathbb{R}$. It is plain that $g$ is also strictly increasing, so representing $\leq$ too, as a new utility function. However, in addition, $g$ is now continuous as regards the usual topology on $\mathbb{R}$. Observe now that $g(t)$ has been obtained from $f(t)$ by subtracting to $f(t)$ the sum of all the jumps of $f$ encountered till we arrive to the point $t$.

This easy Example 1 is, however, quite significative since it lies in the basis of an intuition commonly accepted by many people working in utility theory, namely:

$<<$ If a total preorder $\precsim$ defined on a nonempty set $X$ admits a numerical representation (also known as utility function) $u$, then it also admits another utility function $v$ which is continuous with respect to any natural topology $\tau$ on $X$ and the usual Euclidean topology on the real line $\mathbb{R} .>>$

The reason that supports that intuition is that given a utility function $u$ that represents a total preorder $\precsim$ on a nonempty set $X$, its discontinuities will generate jumps, so that subtracting from $u(x)$ the sum of all the jumps we find before $x$, we would get another utility function $v$, now continuous (exactly as it happens in Example 1).

Thus, we may wonder whether that intuition is correct.

The (surprising) answer is the following: "The above intuition is right. However, the reason that supports it, based on subtracting the jumps before points, is false".

The counterexamples here are neither trivial nor evident. The idea of subtracting sums of jumps works when the number of jump-discontinuities is finite. It also works when those points of discontinuity are isolated. However, a trouble appears when the points of jump discontinuity of a utility function that represents a total preorder $\precsim$ on a nonempty set $X$ constitute a subset $Y \subseteq X$ that is dense-in-itself that is, given $a, b \in Y$ with $a \prec b$, there exists $c \in Y$ such that $a \prec c \prec b$. In that situation, the idea of subtracting the sum of all jumps before points, as before, actually leads to a continuous function, but, unfortunately, that new function could fail to be injective, so that it could no longer be a utility function for $\precsim$. It is noticeable that this insuperable mistake occurs in 
the seminal work by Debreu ([35]). Later, Debreu himself proved in [37], by means of an interesting counterexample, that the argument of subtracting the sum of jumps is wrong, in general, because of measure-theoretic reasons. For a detailed discussion of Debreu's counterexample the reader is referred to Chapter 3 in [44]. As commented in [3], exactly the same flaw has also been a most untoward stumbling block for other scientists. It may indeed be found in several other manuscripts in the classical literature on utility functions, as well as on Entropy and the foundations of Thermodynamics (see, e.g., [18,62-66]). We warn the reader that this mistake appears in Cooper's classical work ([18]) already mentioned in this paper. Let us explain better why the reason that seemed to support our intuition is false (see [3]): To get a continuous entropy function that represents an accessibility relation $\rightsquigarrow$ on a state space $S$, Cooper's argument in [18] is as follows: If we take an entropy function $g: S \rightarrow \mathbb{R}$, by composing it, if necessary, with another increasing function from $\mathbb{R}$ to the open unit interval $(0,1)$, we may assume without less of generality that the given entropy function $g$ is such that $0<g(s)<1$ holds for every $s \in S$. Now, for every $s \in S$, let $\delta_{s}=\sup \{\delta \in \mathbb{R}:(g(s)-\delta, g(s)) \cap g(S)=\varnothing\}$. Similarly, let $v_{s}=\sup \{\delta \in \mathbb{R}:(g(s), g(s)+\delta) \cap g(S)=\varnothing\}$. Then, for all $s \in S$ define $\delta(s)=\delta_{s}$ if $g(s)-\delta_{s} \notin g(S) ; \delta(s)=0$ otherwise, and, similarly, $v(s)=v_{s}$ if $g(s)+v_{s} \notin g(S) ; v(s)=0$ otherwise. It is straightforward to see that the set of points for which either $\delta_{s} \neq 0$ or $v_{s} \neq 0$ is at most countable. Moreover, the sum of all the nonzero $\delta_{s}$ and $v_{s}$ is finite because $g(S)$ is a subset of $(0,1)$. For every $s \in S$, let

$$
h(s)=g(s)-\Sigma_{\{t \precsim s\}} \delta(t)-\Sigma_{\{t \prec s\}} v(t) .
$$

Cooper claims in the main result in his paper that this new function $h$ is a continuous entropy for $\rightsquigarrow$. However, unfortunately, that claim is incorrect in general. Cooper's argument is not valid because, despite the new function $h$ being continuous, it may fail to be injective. To see this, consider the following example: Let $X$ stand for the closed unit interval $[0,1]$, endowed with the usual order $\leq$. Consider an enumeration $\left(q_{n}\right)_{n \in \mathbb{N}}$ of the rational numbers in $[0,1]$, and define the function $g:[0,1] \rightarrow \mathbb{R}$ given by

$$
g(t)=\Sigma_{\left\{n \in \mathbb{N}: q_{n} \leq t\right\}} \frac{1}{n^{2}} .
$$

Then, $g$ is strictly increasing. Moreover, $\delta_{t}=\delta(t)=\frac{1}{n^{2}}$ if $x \in \mathbb{Q} \cap(0,1) ; \delta_{t}=0$ otherwise. In addition, $v_{t}=v(t)=0$ for every $t \in[0,1]$. To conclude, it can now be straightforwardly proved at this stage that the corresponding function $h$ defined as above is a constant, namely $h(s)=0$ holds for every $s \in S$.

At this point, we notice that, to definitively prove that the intuition about the existence of continuous utility representations of representable total preorders was indeed correct, other disparate arguments should be used.

Remark 11. Gerard Debreu found some correct arguments to do so. It is noticeable that ten years elapsed between its former work [35], in which the above discussed mistake appeared, and the correct proof with new arguments was obtained in his paper [54].

The key concept in the new Debreu's approach in [54] was the notion of a gap, and the well-known "Open gap lemma" (see, e.g., Chapter 3 in [44]).

Definition 7. Let $\overline{\mathbb{R}}=\{-\infty\} \cup \mathbb{R} \cup\{+\infty\}$ stand for the extended real line. A degenerate set in $\overline{\mathbb{R}}$ is a set with at most one point. A lacuna of a subset $S \subseteq \overline{\mathbb{R}}$ is a nondegenerate interval that is disjoint from $S$ and has both a lower bound and an upper bound in S. A gap is a maximal lacuna.

Lemma 1. (Debreu's open gap lemma, see [54]) If $S$ is a subset of $\overline{\mathbb{R}}$, there is a strictly increasing function $g: S \rightarrow \overline{\mathbb{R}}$ satisfying that all the gaps of the set $g(S) \subseteq \overline{\mathbb{R}}$ are open. 
Remark 12. Debreu's open gap lemma is indeed a key result in the theory of numerical representations of ordered structures. There are many known proofs of Lemma 1 (see, e.g., Chapters 3 and 4 in [44] for a further account), by authors including Bowen ([67]), Jaffray ([57]), Droste ([68]), Beardon ([69]), Herden and Mehta ([70]), Bosi and Mehta ([71]) and Alcantud et al. ([72]) among others.

As discussed in depth in [44] Chapter 3, if $u$ is a utility function that represents a preorder $\precsim$ on a nonempty set $X$ and $u(X)$ has no half-open, half-closed gaps, then $u$ is continuous with respect to any natural topology for $\precsim$. Thus, if we have just a utility function $u: X \rightarrow \mathbb{R}$ that represents a total preorder $\precsim$, Lemma 1 (Debreu's open gap lemma) comes into our rescue: if we compose $u$ with an increasing function $g: u(X) \rightarrow \mathbb{R}$ whose gaps are open, the composition $v=g \circ u: X \rightarrow \mathbb{R}$ such that $v(x)=g(u(x))$ for every $x \in X$, the new function $v$ is also a utility function that represents $\precsim$. However, in addition, it does not give rise to any half-open, half-closed gap in $v(X)$, so that it is continuous with respect to any natural topology for $\precsim$. Thus, we conclude that the "common intuition" about the existence of continuous representations of total preorders was true. Thus, we can definitively state it as a theorem of the highest importance in the theory of numerical representations of ordered structures.

Theorem 2. Let X be a nonempty set. Let $\precsim$ be a representable total preorder defined on X. Then, $\precsim$ admits a numerical representation that is continuous with respect to any topology on $X$ that is natural with respect to and the usual topology on the real line.

Remark 13. We warn the reader that Theorem 2 is not saying that any possible utility function representing $\precsim$ is continuous. Instead, what Theorem 2 tells us is that, if $\precsim$ is representable, then some continuous numerical representation must exist a fortiori.

Matching Theorems 1 and 2, the following classical results, respectively, due to Eilenberg ([53]) and Debreu ([37]), are immediate consequences.

Corollary 1. Let X denote a nonempty set and $\precsim$ a total preorder on X. Let $\tau$ stand for a natural topology on $X$. The following statements hold true:

(i) (Eilenberg, 1943) If the topological space $(X, \tau)$ is connected and separable, then $\precsim$ admits a utility function which is continuous with respect to the topology $\tau$ on $X$ and the usual topology on $\mathbb{R}$.

(ii) (Debreu, 1964) If the topological space $(X, \tau)$ satisfies the second countability axiom, then $\precsim$ admits a utility function which is continuous with respect to the topology $\tau$ on $X$ and the usual topology on $\mathbb{R}$.

To conclude Section 3, coming back to the axiomatic study of entropy made by Cooper in the spirit of Carathéodory, we conclude that, as stated in the corollary above, Cooper's main result becomes true if we add connectedness to separability, or if we assume second countability instead of the weaker topological hypothesis of separability. Thus, the correct statement is:

$<<$ Suppose that the state space $S$ is given a topology $\tau$ such that the topological space $(S, \tau)$ is either topologically connected and separable or it satisfies the second countability axiom. Assume, in addition, that $S$ is equipped with an accessibility relation $\rightsquigarrow$ which is a total preorder. Suppose also that $\rightsquigarrow$ is continuous as regards $\tau$. Then, there exists a real-valued, continuous, entropy function for the accessibility relation $\rightsquigarrow$ defined on $S .>>$

\section{Mixing and Competition Related to Entropy}

A glance at the classical paper by Cooper [18] shows us a whole section devoted to the analysis of "composition of systems: additivity of entropy" (see also [4]). How could we interpret this concept?

The same situation appears in the much more recent survey by Lieb and Yngvasson (see [19]), namely, they claim that: 
$<<$ For every system there is a function, $S$, on the space of its (equilibrium) states, with the property that one can go adiabatically from a state $X$ to a state $Y$ if and only if $S(X) \leq S(Y)$. This, in essence, is the "entropy principle". The $S$ function can clearly be multiplied by an arbitrary constant and still continue to do its job, and thus it is not at all obvious that the function $S_{1}$ for system 1 has anything to do with the function $S_{2}$ for system 2. Again, we should point out that this is an intuition that is in general false, unless we assume more restrictive conditions ab initio. It is not true, in general, that numerical representations of total preorders, when available, have some sort of linearity. The second remarkable fact is that the $S$ functions for all the thermodynamic systems in the universe can be simultaneously calibrated (i.e., the multiplicative constants can be determined) in such a way that the entropies are additive, i.e., the $S$ function for a compound system is obtained merely by adding the $S$ functions of the individual systems, $S_{1,2}=S_{1}+S_{2}$. To appreciate this fact it is necessary to recognize that the systems comprising a compound system can interact with each other in several ways, and therefore the possible adiabatic transitions in a compound are far more numerous than those allowed for separate, isolated systems. Nevertheless, the increase of the function $S_{1}+S_{2}$ continues to describe the adiabatic processes exactly-neither allowing more nor allowing fewer than actually occur. The statement $S_{1}\left(X_{1}\right)+S_{2}\left(X_{2}\right) \leq S_{1}\left(X_{1}^{\prime}\right)+S_{2}\left(X_{2}^{\prime}\right)$ does not require $S_{1}\left(X_{1}\right) \leq S_{1}\left(X_{1}^{\prime}\right)$. The main problem, from our point of view, is this: What properties of adiabatic processes permit us to construct such a function? To what extent is it unique? What properties of the interactions of different systems in a compound system result in additive entropy functions? $>>$

In our opinion, the idea is to introduce some mathematical background to cope with systems that interact in Thermodynamics, giving rise to a new system. In this way, we should study the relationship (if any) between the entropy of the new system resulting from the interaction and the entropies of the systems we had at hand before. Cooper in [18], as well as Lieb and Yngvasson in [19], also add some axioms in order to deal with this sort of questions. Those additional axioms lean on additivity properties, and the aim here is finding entropy functions that are unique up to linear transformations. In a similar way, a battery of papers by Klimenko (see [38,73-77]) deals with concepts as mixing (see also [78]) and competition of systems. It is not our intention here to make a criticism on those papers. However, to the eyes of a mathematician, it is difficult to isolate the definition of the concepts "mixing" and "competition". Perhaps the idea of what is an abstract definition is totally different for mathematicians, physicists, chemists, engineers, economists or psychologists. It is quite typical that, instead of giving a rigorous abstract (mathematical) definition of a concept, some authors describe properties of the item they are analyzing. However, this provokes that the non-specialist (who, in particular, sometimes could be the pure mathematician), searching for a definition, is forced to make some interpretations, putting everything in a more abstract setting. Here are a few examples in which to draw a clear definition of what "mixing" and/or "competition" really is, constitutes a hard task for an interested but non-specialist researcher.

(1) $<<$ Mixing is commonly understood as blending of two (or more) gases or liquids until a uniform mixture is achieved. Mixing preserves the identity of participating molecules and is thus different from chemical reactions. If molecules have little interactions, the resulting mixture is called an ideal mixture and characterized by maximal possible disorder and maximal possible entropy. Alternatively, interactions between molecules can reduce molecular chaos and the entropy of mixing falls below its maximal value. $>>$

(2) $<<$ The process of mixing can be simulated by a sufficiently large number of notional particles, randomly walking from the region of their initial confinement and diffusing to occupy the whole domain. The fundamental similarity between diffusion (mixing) and random walks was established first in physics and then in mathematics. $>>$

(3) <<Typical processes of mixing are such that several systems of different composition, that are initially independent and separated one another, and such that each of them is in a thermodynamic state of chemical equilibrium, are mixed without chemical reaction. This is done by removal of the impermeable barriers and partition that separated the systems ab 
initio. After a time, a new thermodynamical state of internal equilibrium is reached in the new closed system. $>>$

(4) $\quad<<$ The idea of abstract competition was derived from the long-standing tradition of modeling turbulent combustion. In these models, it is common to use Pope particles (i.e., notional particles with properties and mixing). If conventional mixing is replaced by competitive mixing, these notional particles may be seen and used as computational incarnations of generic elements engaged in abstract competition. Competitive mixing can be deployed to characterize various processes: turbulent combustion, invasion waves and other related phenomena. Unlike conventional conservative mixing, competitive mixing can display complex behavior with sophisticated interdependencies, which is of special interest for this review. $>>$

(5) $<<$ Competitive systems involve the process of competition in its most generic form. The elements of competitive systems compete with each other according to preset rules. The rules define the winners and losers for each competition round based on properties of the elements. The properties of the losers are lost while the winners utilize the resource vacated by the losers and duplicate their properties. The process of duplication is not perfect and involves random mutations, which are mostly detrimental for the competitiveness of the elements. $>>$

(6) $<<$ Competitive systems, which are typically associated with complex stochastic behavior, are common in the real world. Abstract competition, which studies generic principles of competition in their most abstract form, can be interpreted as a form of mixing. This mixing, which is called competitive mixing, can be used to characterize various processes: turbulent combustion, invasion waves and other related phenomena. Unlike conventional conservative mixing, competitive mixing can display complex behavior with sophisticated interdependencies. $>>$

Remark 14. The previous paragraphs of Section 4 try to set and discuss a topic related to chemical potential that arose from exchange particles between systems. To the eyes of physicists, that concept can be described through Gibbs grand canonical ensemble theory (see $[25,79,80])$. However, mathematicians may still think that a further step in rigor is necessary here, therefore looking for clear definitions, at least in the mathematical background level on which that theory leans.

Let us do here a mathematical interpretation to fix ideas:

In our opinion, the key mathematical framework on which this kind of questions could be settled and analyzed is that of algebraic ordered structures. That is, when we are given an ordered structure (e.g., a nonempty set $X$ endowed with a total preorder $\precsim$ ), it may happen that $X$ also has some additional structure of algebraic stuff that, hopefully, could be compatible with the ordering involved. To put an example, in the lexicographic plane $\left(\mathbb{R}^{2}, \preceq_{L}\right)$, we may consider an operation $\bar{\mp}$ of coordinatewise addition or sum of vectors, given by $(x, y) \overline{+}(z, t)=(x+z, y+t)$ for all $x, y, z, t \in \mathbb{R}$. Now, we may notice that there is a compatibility or interaction between the total order $\preceq_{L}$ and the algebraic binary operation $\bar{\mp}$, namely $(x, y) \prec_{L}(z, t) \Leftrightarrow(x+a, y+b)=(x, y) \mp(a, b) \prec_{L}(z+$ $a, t+b)=(z, t) \mp(a, b)$, for every $(x, y),(z, t),(a, b) \in \mathbb{R}^{2}$. In the case when an algebraic operation is compatible with the ordered structure, it is typical and natural to search for the existence of numerical representations (e.g., utility functions, entropies) that not only preserve the ordered structure, but they are algebraic homomorphisms too, say, on the additive real line $(\mathbb{R},+)$. For instance, if $X$ is a nonempty set endowed with a total preorder $\precsim$ and a binary operation, say $*$, such that $x \precsim y \Leftrightarrow x * a \precsim y * a$ holds true for every $x, y, a \in X$, we could be interested in finding a numerical representation $u$ for $\precsim$ such that, in addition, it also accomplishes that $u(x * y)=u(x)+u(y)$ for every $x, y \in X$.

As commented in depth, e.g., in [81], in Mathematics two different but finally converging approaches could lead to the study of algebraic ordered structures. On the one hand, as in the present paper, we encounter some ordered structure where it also arises an extra algebraic operation, compatible in some way with the ordering. In this former situation, the starting point is the ordered structure, and the algebra appears (last but not least) as something additional. On the other hand, we 
may deal with algebraic structures (e.g., a group, a semigroup, and a vector space) that are endowed with some kind of ordering that is compatible in some way with that, given at origin, algebraic structure. In other words, in this second situation, we begin with an algebraic structure, and the ordering is something additional, but not of minor importance.

Coming back to the entropy setting in Thermodynamics, we could understand "mixing" as some kind of interaction between systems. That is, if $S$ is the state system, two different systems $s, t \in S$ could interact in some way giving rise to a new system $r \in S$. Thus, it seems natural to understand the interaction as a binary operation, say o, on the set $S$ (i.e., $\circ: S \times S \rightarrow S$ ), and consequently write $s \circ t=r$. Depending on the properties of the interaction $\circ$, and assuming that an accessibility relation $\rightsquigarrow$ is defined on $S$, we could be interested in finding an entropy function $f$ that not only represents $\rightsquigarrow$ but, in addition, it satisfies that $f(s \circ t)=f(s)+f(t)$ holds true for every $s, t \in S$.

With respect to "competition", we can also understand that notion as arising under the same mathematical setting as the aforesaid for mixing. Let us consider an example not coming from Thermodynamics or entropy, but clearly equivalent to situations as reactions, combustion, etc., where a component, the loser, is absorbed (or vanished, or even neglected) by another one, the winner: Thus, suppose that an animal (e.g., a lion) is a predator and another one (e.g., a gazelle) is a prey. If both animals meet and fight, and the lion kills the gazelle and eats it, we may interpret this as a situation in which we start with a lion plus a gazelle, and we end just with one lion (perhaps stronger since it has got its meal) and no gazelle. If we represent this "fight", by means of a suitable mathematical abstraction, as an operation $\circ$ defined on the animal world, and $l$ (respectively, $g$ ) denotes the lion (respectively, the gazelle), we would write something as $l \circ g=l$. Similar situations appear in Thermodynamics when systems interact or compete in a way that one of them is a winner, the other is a loser, and the final result is that the first system survives (maybe with richer properties) while the second disappears. In any case, they could also be mathematically interpreted by means of a state system $S$ where some binary operation $\circ$ is defined, eventually allowing cases as $s \circ t=s$ for some $s, t \in S$. Whenever $S$ is endowed with an accessibility relation $\rightsquigarrow$, we may also be interested in finding entropy functions that also could, in some way, give further information about the "competition" operation $\circ$ on $S$.

Therefore, we may conclude this discussion by saying that we should pay attention to ordered structures endowed with some compatible algebraic operation, and look for numerical representations (if any) that not only preserve the ordered structure, but also the algebraic one as well.

At this stage, another important fact is to select the suitable algebraic structure to deal with. In this sense, we point out that, in the literature on numerical representations of ordered structures (mainly, total preorders) endowed with some compatible algebraic operation, one may encounter, mainly, classical results on ordered groups and semigroups (see, e.g., [81-104]). A key reference here is the book by L. Fuchs [105] However, there are also deep studies on lattices, where a famous reference is the book [106] by Garret Birkhoff (see also [107]), ordered vector spaces (see, e.g., [108-112]) and even real algebras (see, e.g., [56]).

To go ahead in our study, to choose an adequate algebraic setting, it still seems necessary to observe some features of the systems we want to analyze then. Thus, let us assume that we have in mind the idea of a thermodynamical approach related to mixing or competition processes, so that the state space $S$ is equipped with some binary operation, say o. In fact, such operation represents "mixing", being understood, so-to-say, as the removal of the impermeable barriers that separated two given systems $s, t \in S$, so allowing them to a composition, an interaction or competition (e.g., a chemical reaction). Thus, we think that the most suitable starting point here is assuming that $(S, \circ)$ is a semigroup (see Definition 8). As already commented in [1], it seems more plausible to interpret the state space as a semigroup rather than a group, because there are many chemical reactions that cannot be reverted, so that the concept of a "converse element" that would be compulsory to deal with the mathematical structure of an algebraic group could be nonsensical in this approach. However, the idea of the mixing (composition and reaction) being associative seems to be more suitable: if we admit that the composition of systems by mixing is a binary operation $\circ$ defined on $S$ (understood as the collection 
of all possible systems), it is quite natural to assume that the operation $\circ$ is associative (and perhaps commutative, too).

Remark 15. It is noticeable that these conditions were not commented in Section 4 in [18] nor (more than thirty years later) in the survey [19], despite of being implicitly assumed or taken for granted. In [18] some new axioms were added to the Carathéodory setting to deal with entropy functions that also preserve sone algebraic structure. The use of axioms to deal with numerical representations of, say, total preordered sets endowed with some compatible algebraic structure, in a way that the representation or utility function is an algebraic homomorphism (usually called additive) was launched in a pioneer work by O. Holder (see [82,105]). Since then, it has also appeared in several other multidisciplinary settings, e.g., the theory of extensive measurement in Mathematical Psychology (see, e.g., [96,101]). However, its mathematical background is exactly the same as the one encountered when dealing with "additive entropies" in Thermodynamics, as in $[18,19,113,114]$.

By the way, Cooper's approach in [18] is actually more sophisticated, since he considers n-ary operations (instead of just a binary operation o) to cope with composition of systems. However, we might still assume, at least implicitly, that the special kind of n-ary operations considered in [18] could be decomposed in a way as an ad hoc iteration of binary compositions.

Definition 8. A semigroup is an algebraic structure $(S, O)$ that consists of a nonempty set $S$ endowed with an internal binary operation $\circ$ that is associative. If, in addition, $S$ has a null element e such that $s \circ e=e \circ s=s$ holds true for every $s \in S$, then $(S, O)$ is said to be a monoid. Furthermore, if each element $s$ of a monoid $(S, O)$ has an inverse $s^{*}$ such that $s \circ s^{*}=s^{*} \circ s=e$, then $(S, \circ)$ is said to be a group. A semigroup $(S, \circ)$ endowed with a total preorder $\precsim$ is said to be a totally preordered semigroup if there is a compatibility between the total preorder $\precsim$ and the binary operation $\circ$ such that $s \precsim t \Leftrightarrow s \circ u \precsim t \circ u \Leftrightarrow u \circ s \precsim u \circ t$ holds true for every $s, t, u \in S$. (This last property is also known as the translation-invariance of the total preorder $\precsim$ as regards the binary operation $\circ)$. Notice that, in particular, a totally preordered semigroup is always cancellative, namely $s \circ u=t \circ u \Leftrightarrow s=t \Leftrightarrow u \circ s=u \circ t(s, t, u \in S)$. A totally preordered semigroup $(S, \circ, \precsim)$ is said to be positive (respectively, negative) if $x \prec x \circ x$ (respectively, if $x \circ x \prec x$ ) holds true for every $x \in S$. In addition, an element $t \in$ is said to be positive (respectively, negative) if $t \prec t \circ t$ (respectively, if $t \circ t \prec t$ ) holds. If $(S, \circ, \precsim)$ is a totally preordered semigroup, its subset $S^{+}=\{s \in S: s \prec s \circ s\}$ (respectively, $S^{-}=\{s \in S: s \circ s \prec s\}$ ) is said to be the positive cone (respectively, the negative cone) of $(S, \circ, \precsim)$. Notice that, when $S$ is a totally preordered monoid, an element $s \in S$ is positive (respectively, negative) if and only if $e \prec s$ (respectively, if $s \prec e$ ) holds, where e stands for the null element of the monoid.

Remark 16. If $(S, \circ, \precsim)$ is a totally preordered semigroup, where $\precsim$, we might pass to be working with a quotient space $S / \sim$ whose elements are the indifference classes of the elements of $S$ with respect to $\sim$. That is, given $s \in S$, its corresponding class $[s] \in S / \sim$ is the set $\{t \in S: t \sim s\}$. Since there is a compatibility between the total preorder $\precsim$ and the binary operation, the quotient $S / \sim$ inherits a structure of totally ordered semigroup by considering in a natural way that the binary operation $\circ$ as well as $\precsim$ directly act on the indifference classes that $\sim$ induces on $S$.

By this reason, henceforward we work with totally ordered semigroups, instead of totally preordered semigroups, unless otherwise stated.

Definition 9. A totally preordered semigroup $(S, \circ, \precsim)$ is called additively representable if there exists a real-valued function $u: S \rightarrow \mathbb{R}$ satisfying that $u(s \circ t)=u(s)+u(t)$ and also $s \precsim t \Leftrightarrow u(s) \leq u(t)$ hold true for every $s, t \in S$. A map $u$ with these properties is said to be an additive utility function.

To characterize the totally ordered semigroups that are additively representable, we still need to introduce the following definition. 
Definition 10. A positive semigroup $(S, \circ, \precsim)$ is said to be Archimedean if for every $s, t \in S$ with $s \prec t$, there exists a natural number $k \in \mathbb{N}$ such that $t \prec s^{k}=s \circ \ldots(k-$ times $) \ldots \circ s$. Moreover, it is called super-Archimedean if for every $s, t \in S$ such that $s \prec t$ there exists a natural number $k \in \mathbb{N}$ such that $s^{k+1} \prec t^{k}$.

In the case of a totally ordered group, a classical result coming from Algebra, and stated by Hölder early in 1901, characterizes the additive representability in terms of Archimedeaness, as follows (see [82] or [106], p. 300).

Proposition 1. A totally ordered group $(G, 0, \precsim)$ is additively representable if and only if its positive cone is Archimedean.

In the case of a totally ordered semigroup, the additive representability was characterized by N. G. Alimov in 1950, in terms of the super-Archimedean property, as follows (see [94,97] or the reference-note 21 on p. 26 in [12]).

Proposition 2. A totally ordered semigroup $(S, \circ, \precsim)$ is additively representable if and only if its positive cone $S^{+}$is super-Archimedean and its negative cone $S^{-}$is also super-Archimedean when endowed with the transpose order $\precsim^{t}$ given by $s \precsim^{t} u \Leftrightarrow u \precsim s \quad(s, u \in S)$.

Remark 17. (i) The property of Archimedeaness on positive totally ordered semigroups does not suffice to guarantee the existence of an additive utility function. An example is the semigroup $S=(0+$ $\infty) \times(0,+\infty)$, equipped with the lexicographic total order and the binary operation $(a, b) \circ(c, d)=$ $(a+c, b+d)$. As already commented in Section 3, this ordered set is not representable, even if we do not pay attention to additivity (see, e.g., [106], pp. 200-201).

(ii) A positive super-Archimedean totally ordered semigroup $(S, 0, \precsim)$ is, in particular, Archimedean. (For a detailed proof, see, e.g., [97].) However, the converse claim is not true in general: indeed $S=(0+$ $\infty) \times(0,+\infty)$ endowed with the lexicographic ordering $\preceq_{L}$ is not super-Archimedean since, for instance, $(2,2) \prec_{L}(2,8)$ but $(2 k, 8 k) \prec_{L}(2 k+2,2 k+2) \quad(k \in \mathbb{N})$.

(iii) If $(G, \circ, \precsim)$ is an Archimedean totally ordered group, its positive cone is Archimedean and also its negative cone is Archimedean with respect to the transpose order (see, e.g., [97] for a proof and further details, as well as related properties).

Back to the consideration of a thermodynamical setting related to a mixing process, we may summarize the previous facts by claiming that, for the existence of an additive entropy the following extra axioms should be added to the Carathéodory ones, in the spirit of Section 4 in [18]:

(i) The phase system $S$, endowed with the mixing operation $\circ$ and the accessibility relation $\rightsquigarrow$ must be a totally preordered semigroup.

(ii) Both the positive cone, and the negative cone endowed with the transpose $\rightsquigarrow^{t}$ of the accessibility relation, of the totally ordered semigroup structure $(S, \circ, \rightsquigarrow)$, must be super-Archimedean.

To complete the panorama, and following the set of ideas in [1], we should also observe that a totally ordered group $(S, \circ, \precsim)$ could also be endowed with some topology $\tau$. In that case, it is natural to search for the existence of additive utility functions that are continuous with respect to the topology $\tau$ on $S$, and the usual Euclidean topology on the real line. Thus, provided that some topology $\tau$ is at hand, we could deal with ideas related to limits, proximity or continuity and related items.

Remark 18. In the thermodynamical setting, questions of this kind, namely the existence of continuous and additive entropies, were already considered in Section 4 of Cooper's key paper [18].

Let us analyze now the main results arising in the mathematical background of this approach. 
Remark 19. Even being representable by an additive utility function, a totally ordered semigroup could fail to admit a continuous and additive representation. An example is the semigroup $Z=[2,3) \cup[4,+\infty) \subset \mathbb{R}$ with the usual sum and order of the reals as binary operation, and equipped with the relative topology inherited from the usual one in $\mathbb{R}$. It can be proved (see, e.g., [98,99]) that the main reason for the non-existence of an additive and continuous representation is the discontinuity with respect to the order topology, of the sum in $\mathrm{Z}$.

Definition 11. A topological semigroup $(S, \circ, \tau)$ is a semigroup $(S, \circ)$ equipped with a topology $\tau$ that is related to the binary operation $\circ$ in the following sense: it makes continuous the binary operation $\circ: S \times S \rightarrow S$ that maps the pair $(s, t) \in S \times S$ to the element $s \circ t \in S$. (Here, on $S \times S$ we consider the product topology coming from $\tau$ on $S$.)

A totally ordered semigroup $(S, \circ, \precsim)$ is called a topological totally ordered semigroup when $\circ$ is continuous with respect to the order topology $\tau_{\precsim}$ on $S$ (and its corresponding product topology on $S \times S$ ).

A topological group is defined as a group $(G, 0)$ endowed with a topology $\tau$ such that the binary operation $\circ$ is continuous, and also the unary operation of taking inverses in $G$, that assigns to each element $s \in G$ its inverse $s^{*}$ is also continuous with respect to the topology $\tau$ in $G$.

Remark 20. A famous result, extremely important in this approach, is due to Nyikos and Reichel (see [87]) and states that any totally ordered group is topological with respect to the order topology. It actually proves that on a totally ordered group $(G, \circ, \precsim)$, both the operation $\circ$ and the unary operation of taking an inverse are, a fortiori, continuous as regards the order topology $\tau_{\precsim}$. Notice that this is indeed a theorem about "automatic continuity", in the sense that the compatibility between the total order $\precsim$ and the algebraic operation $\circ$ already carries the continuity of the group operations, namely o and the unary one of taking inverses, with respect to any natural topology for $\precsim$ defined on $G$.

However, the analogous result for semigroups is not valid in general. (The counterexample shown in Remark 19 also fits well here.)

The following result, whose proof appears in [98], is perhaps the best one that one could expect to find in this approach.

Theorem 3. Let $(S, \circ, \precsim)$ be a topological totally ordered semigroup. Assume also that $S$ is endowed with a topology $\tau$ that is natural with respect to the linear order $\precsim$. Suppose also that the positive cone $S^{+}$is super-Archimedean with respect to $\precsim$, whereas the negative cone $S^{-}$is super-Archimedean with respect to the transpose order. Then, $S$ is representable by an additive utility function $u: S \rightarrow \mathbb{R}$ such that $u(s \circ t)=$ $u(s)+u(t)$ and $s \precsim t \Leftrightarrow u(s) \leq u(t)$ hold true for every $s, t \in S$, and, in addition, the map $u$ is continuous with respect to the given topology $\tau$ in $S$ and the usual one in $\mathbb{R}$.

To finish Section 4, coming back once more to the thermodynamical approach, we may draw the conclusion that, for the existence of a continuous additive entropy. we could add the following suitable extra axioms to the Carathéodory-Cooper's setting analyzed in Section 4 of Cooper's paper [18]:

(i) The state space $S$, equipped with the mixing operation $\circ$ and the accessibility relation $\rightsquigarrow$ should be a totally ordered semigroup.

(ii) Both the positive cone, and the negative cone endowed with the transpose $\rightsquigarrow^{t}$ of the accessibility relation, of the totally ordered semigroup $\operatorname{structure}(S, \circ, \rightsquigarrow)$, should be super-Archimedean.

(iii) $S$ should be endowed with a topology $\tau$ that is natural with respect to the accessibility relation $\rightsquigarrow$. (In other words: $\precsim$ should be continuous as regards $\tau$.)

(iv) The structure $(S, \circ, \rightsquigarrow)$ should be a topological totally ordered semigroup. (That is, $\circ$ should be continuous with respect to the order topology $\tau_{\precsim}$ on $S$ and the product topology on $S \times S$.) 


\section{Representability of Interval Orders and Semiorders}

Again, we follow some ideas already launched e.g., in [1]. To start with, we define the representability of interval orders. Since the representability of a binary relation $\mathcal{R}$ on a nonempty set $X$ through a function $u: X \rightarrow \mathbb{R}$ such that $x \mathcal{R} y \Leftrightarrow u(x) \leq u(y)$ forces $\mathcal{R}$ to be a total preorder, as already commented at the beginning of Section 3 , the numerical representations of other kinds of orderings (as interval orders or semiorders) compulsorily need some variation in the way we represent them. In the case of interval orders, the classical representations (when available) use two real-valued functions instead of just one, as stated in the next Definition 12.

Definition 12. Let $X$ stand for a nonempty set. An interval order $\prec$ defined on $X$ is called representable (as an interval order) if there exists a pair $(u, v)$ of real-valued functions $u, v: X \longrightarrow \mathbb{R}$ accomplishing that $x \prec y \Leftrightarrow v(x)<u(y) \quad(x, y \in X)$. The pair $(u, v)$ is called a utility pair representing $\prec$, also known as a numerical representation of the given interval order $\prec$.

Remark 21. Notice that since an interval order $\prec$ is an asymmetric binary relation on $X$, if it is representable by means of a pair $(u, v)$ then we have a fortiori that $u(t) \leq v(t)$ holds for every $t \in X$. This gives rise to the following interpretation, that quite probably inspired the term of an interval order in the specialized literature. This term is attributed to Peter C. Fishburn (see [42,43]): Whenever the interval order $\prec$ admits a numerical representation by means of a pair $(u, v)$, each element $t \in X$ can be put in direct relationship with an interval $[u(t), v(t)]$ of the real line. (Observe also that such interval could degenerate to a single point if $u(t)=v(t)$.) Here, $a \prec b$ means that the interval $[u(a), v(a)]$ is located on the real line $\mathbb{R}$ totally on the left of the interval $[u(b), v(b)]$ without touching it.

To characterize the numerical representability of interval orders, we introduce now more necessary definitions.

Definition 13. Associated to an interval order $\prec$ defined on a nonempty set $X$, we shall consider two new binary relations.

These binary relations are said to be the traces of $\prec$. They are, respectively, denoted by $\prec *$ (left trace) and $\prec^{* *}$ (right trace), and defined as follows: $x \prec^{*} y \Leftrightarrow x \prec z \precsim y$ for some $z \in X$, and similarly $x \prec * * y \Leftrightarrow x \precsim z \prec y$ for some $z \in X(x, y \in X)$.

Remark 22. We denote $x \precsim^{*} y \Leftrightarrow \neg\left(y \prec^{*} x\right), \quad x \sim^{*} y \Leftrightarrow x \precsim^{*} y \precsim^{*} x$, and $x \precsim^{* *} y \Leftrightarrow \neg\left(y \prec^{* *} x\right)$ and $x \sim^{* *} y \Leftrightarrow x \precsim^{* *} y \precsim^{* *} x \quad(x, y \in X)$. Notice that the binary relation $\precsim^{*}$ (respectively, $\left.\precsim^{* *}\right)$ is the adjoint of $\prec^{*}$ (respectively, of $\prec^{* *}$ ). Both of these adjoints are indeed total preorders on X. Moreover, the indifference relation $\sim$ associated to the interval order $\prec$ is transitive if and only if $\precsim^{*}, \precsim^{* *}$ and $\precsim$ coincide. In this case, $\precsim$ is actually a total preorder on X. The converse is also true, and all these claims are also equivalent to say that the associated symmetric binary relation $\precsim$ is transitive (see, e.g., [45] as well as Proposition 1.6 .11 in [44] for further information).

Definition 14. Let $X$ be a nonempty set. An interval order $\prec$ defined on $X$ is said to be interval order separable if there exists a countable subset $D \subseteq X$ such that for every $x, y \in X$ with $x \prec y$ there exists $d \in D$ such that $x \precsim * d \prec y$.

The following key fact, that characterizes the numerical representability of interval orders is well-known in this literature (for a detailed proof, as well as other equivalent results, see, e.g., [45]).

Theorem 4. An interval order $\prec$ defined on a nonempty set $X$ is representable if and only if it is interval order separable. 
Remark 23. Other characterizations, that use a more sophisticated set of conditions that are equivalent to the representability of an interval order, had already been obtained earlier by Fishburn in 1973 (see [115]) and, independently, by Doignon et al. in 1984 (see [116]).

In the case of semiorders, apart from its possible representation just as interval-orders as stated in Definition 12, the classical numerical representations (when they exist) consist of a real-valued function plus a strictly positive constant.

Definition 15. Let $\prec$ denote a semiorder defined on a nonempty set $X$. We say that $\prec$ is representable in the sense of Scott and Suppes (see $[40,117]$ ) when there is a real-valued function $u: X \rightarrow \mathbb{R}$ and a strictly positive constant $k>0$ such that $a \prec b \Leftrightarrow u(a)+k<u(b)$ holds true for every $a, b \in X$. The pair $(u, k)$ is then said $a$ Scott-Suppes pair representing $\prec$.

Remark 24. If the semiorder $\prec$ is representable in the sense of Scott and Suppes by means of the pair $(u, k)$, it is also Scott-Suppes representable by the pair $(v, 1)$, where $v: X \rightarrow \mathbb{R}$ is defined by $v(t)=\frac{u(t)}{k}(t \in X)$. In other words, in the definition of Scott-Suppes representability of a semiorder, we can take $k=1$ without loss of generality.

At this stage, notice once more that " $k$ " acts here as a threshold of discrimination. In fact, in suitable settings it could also be interpreted as a "quantum".

To characterize the Scott-Suppes representability of semiorders, we need to introduce the next key definition.

Definition 16. Let $X$ be a nonempty set. A semiorder $\prec$ defined on $X$ is called regular with respect to sequences if there are no $x, y \in X$ and sequences $\left(x_{n}\right)_{n \in \mathbb{N}},\left(y_{n}\right)_{n \in \mathbb{N}}$ of elements in the set $X$ such that $x \prec \ldots \prec x_{n+1} \prec$ $x_{n} \prec \ldots \prec x_{3} \prec x_{2} \prec x_{1}$ happens nor, dually, $y_{1} \prec y_{2} \prec y_{3} \prec \ldots \prec y_{n} \prec y_{n+1} \prec \ldots \prec y$ holds true. In other words, the given set $X$ never contains, as regards the given semiorder $\prec$, any infinite up chain with an upper bound, nor else any infinite down chain with a lower bound.

The Scott-Suppes representability of semiorders is finally characterized as follows (see, e.g., [117-120]):

Theorem 5. A semiorder $\prec$ defined on a nonempty set $X$ is representable in the sense of Scott and Suppes if and only if it is interval order separable and regular with respect to sequences.

Remark 25. The obtention of a characterization of the representability of semiorders by means of conditions that, as the ones in the statement of Theorem 5 has been a truly "tour de force". It was posed as an open problem in $[39,40,121]$ among other classical papers. Since then, several other characterizations were achieved, some of them only valid for particular cases (see, e.g., [118,122-126]).

A next step here would be the analysis of the continuous representability of interval orders and semiorders, provided that the nonempty set $X$ on which an interval order or a semiorder $\prec$ has been defined is also endowed with some topology $\tau$. Unfortunately, in these cases (namely interval orders and semiorders) an analogous result to the famous Debreu's open gap lemma (see Lemma 1 in Section 3 above) is, in general, no longer available (see, e.g., [127]). In fact, in the case of semiorders, the analogous of the Debreu's open gap lemma is false in general, as proved in Example 1 and Remark 7 in [128].

Unlike the case of total preorders, a characterization, valid for the general case, of the continuous representability of interval orders is not known yet. The same happens for semiorders.

At this stage, we need to introduce some mathematical background about the continuous representability of interval orders. 
Definition 17. Let $X$ be a nonempty set equipped with a topology $\tau$ and an interval order $\prec$. The topology $\tau$ is said to be natural as regards $\prec$ if, for all $x \in X$, the sets $\{t \in X: t \prec x\},\{t \in X: x \prec t\},\{t \in X: t \prec *$ $x\},\left\{t \in X: x \prec^{*} t\right\},\left\{t \in X: t \prec^{* *} x\right\}$ and, finally, $\left\{t \in X: x \prec^{* *} t\right\}$ are all open sets in the topology $\tau$.

When we are given an interval order $\prec$ on a topological space $(X, \tau)$ in which the topology $\tau$ is natural, the following important partial answer the problem of characterizing the continuous representability of an interval order $\prec$ on a topological space $(X, \tau)$ arises. The proof of this important result may be seen in [46], Theorem 1 on pp. 310-312.

Theorem 6. Let X be a nonempty set endowed with a topology $\tau$. Let $\prec$ be an interval order on $X$. Assume also that the topology $\tau$ is natural with respect to the interval order $\prec$. The following statements are equivalent:

(i) The interval order $\prec$ is interval order separable.

(ii) The interval order $\prec$ is representable through a pair $(U, V)$ of continuous real-valued functions such that, in addition, $U$ represents the total preorder ${ }^{* *}$ and $V$ represents the total preorder $\precsim *$.

Remark 26. As far as we know, the result stated in Theorem 6 is the best one on the continuous representability of interval orders obtained until now. Nevertheless, Theorem 6 is only a partial answer to the still unsolved problem of characterizing the continuous representability of an interval order. This is due to the fact that nothing has been said about the possibility of the existence of a pair $(u, v)$ of continuous functions representing $\prec$ but such that either $u$ does not represent ${ }^{* *}$ or $v$ does not represent $\precsim^{*}$. In this direction, some further discussion can be seen in Section 3 in [129], where other (still partial) solutions to the problem of characterizing the continuous representability of interval orders have been achieved. These are based on topological conditions different from the ones involved in the main results launched in [46]. Concerning the continuous representability of semiorders, in [128] some partial characterizations have recently been obtained, too.

To conclude Section 5, we still should pay attention to the representability of interval orders and semiorders defined on a nonempty set $X$ where some additional algebraic structure (e.g., a semigroup) appears, and, in some sense, it is compatible with the ordering.

However, and perhaps to our surprise, the existence of additive representations of an interval order $\prec$ defined on a nonempty set $X$ may actually force the interval order to degenerate, so that its adjoint is a total preorder, as next Theorem 7 shows.

Theorem 7. Let $S$ be a nonempty set endowed with an associative binary operation $\circ$. (That is: the structure $(S, \circ)$ is a semigroup.) Let $\prec$ be an interval order defined on $S$. Assume that $\prec$ is compatible with $\circ$ in the following sense: $a \prec b \Leftrightarrow a \circ c \prec b \circ c \Leftrightarrow c \circ a \prec c \circ b$ holds true for every $a, b, c \in S)$. Then, if the interval order $\prec$ admits a representation by means of a pair $(u, v)$ such that, in addition, both $u$ and $v$ are additive (i.e., $a \prec b \Leftrightarrow v(a) \prec u(b) ; u(a \circ b)=u(a)+u(b) ; v(a \circ b)=v(a)+v(v)$ hold true for every $a, b \in S)$, then $\precsim$ is indeed a total preorder. In particular, both $\precsim$ and $\sim$ are transitive.

Proof. See Theorem 3 in [1].

\section{Intransitive Processes}

Coming back to the thermodynamical approach, we pay attention to the reference [73] as well as to Section 3 in [1].

Most of the studies that we include in Section 6 had already appeared in [1]. However, since our intention is to furnish, throughout the present manuscript, a survey of results concerning the mathematical foundations of Entropy, we have decided to include them again in Section 6 for the sake of completeness.

Thus, already in the abstract of the paper [73], one reads that: 
$<<$ While conventional thermodynamics is constrained by its zeroth law and is fundamentally transitive, the transitivity of competitive thermodynamics depend on the transitivity of the competition rules. Intransitivities are common in the real world and are responsible for common behavior in competitive systems. $>>$

With similar ideas in mind, in [38], it is claimed that:

$<<$ While physical thermodynamics is always transitive, the same cannot be stated a priori about apparent thermodynamics. $>>$

Therefore, it is interesting at this stage to find a suitable mathematical approach in order to interpret intransitivities arising in state spaces.

A possible mathematical setting that may help us to do so was already introduced in the appendix of the reference [73]. Nevertheless, neither in that study nor in the more recent [38], the key notion of an interval order just defined and analyzed in Sections 2 and 5 of the present manuscript has been used as a tool.

In our opinion, as already shown in [1], to deal with intransitivities, the concept of an interval order is the key notion and the results on interval orders should be considered as the main device.

Remark 27. In fact, although they were already implicit in Wiener's works (see [41]) under a different terminology, it is commonly accepted that interval orders were (re)-introduced later to understand better typical situations that arise in Psychology (Measurement Theory) or Economics (Utility Functions), where some intransitivities related to the concept of a preference were encountered in the corresponding models (see, e.g., $[39,40,42,43,130])$. Remember here that the indifference relation $\sim($ or, equivalently, the relation $\precsim)$ associated to an interval order $\prec$ defined on a nonempty set $X$ is transitive if and only if $\precsim^{*}, \precsim^{* *}$ and $\precsim$ coincide, and in this case $\precsim$ is actually a total preorder on X (see Definition 13).

By the way, an interval order $\prec$ is always transitive. Only its associated relations $\sim$ and $\precsim$ could fail to be transitive.

Example 2. In cases in which $\sim$ is not transitive, it is typical that some situations arising in, say, experiments or processes can be interpreted as paradoxical, so-to-say. For instance, in theoretical computer science, when several computers are working in distributed computation, we may pay attention, given a task $x$, to the interval of time $[s(x), f(x)]$ in which the task $x$ is being executed by the system of computers, so that $s(x)$ (respectively, $f(x)$ ) is the moment when the task $x$ starts (respectively, finishes). Therefore, we can assign to each task its corresponding interval of time, and accordingly declare that a task $x$ is "totally precedent" with respect to another task $y$, if $f(x)<s(y)$. This precedence relation that we may denote $\prec$, is, obviously, a representable interval order. When two intervals corresponding to different tasks overlap, there are moments of time in which we could see both tasks being active (at the same time), so that we could interpret them as "simultaneous". This corresponds to the associated relation $\sim$, which is not transitive in this case. The same occurs with the associated binary relation $\precsim$, so that if we take $\precsim$ (instead of the more restrictive $\prec$ ) as a binary relation that expresses also an idea of "precedence" (but weaker than before), the intransitivity of $\precsim$ sounds as something "paradoxical".

In fact, intransitivities of this kind can also occur in physical processes and/or chemical reactions: Thus, as already mentioned in [73], we have that:

$<<$ Intransitivities are possible in chemical reactions. The Belousov-Zhabotinsky reaction is known to display a cyclic chemical behavior in a homogeneous mixture, which is unusual since most chemical kinetics tend to monotonically converge to an equilibrium or steady state. (...) The simplest chemical kinetic scheme that can adequately simulate the Belousov-Zhabotinsky reaction is Oregonator [131]. This scheme involves an essential intransitivity. $>>$

Remember that, if the accessibility relation of the state space is a total preorder, this kind of intransitivities would never occur.

Moreover, as explained in [73]:

$<<$ The choice in favor of considering intransitivities needs to be made not because intransitivity can immediately explain all the complexities of the surrounding world but because transitive 
competition certainly cannot. This does not prevent many specific features from having perfectly transitive explanations, while intransitivity is associated with more complex effects. (...) It is worthwhile, however, to draw the attention of the reader to the immediate unresolved problems associated with intransitive behavior.>>

In addition, in [76], the author claims that:

$<<$ When treated simplistically, intransitivity may seem to be illogical but, nevertheless, it is common in nature and needs to be studied. $>>$

Taking into account these last comments encountered in the aforementioned references, as well as the study made in Section 3 in [1], we can give some answers to the problem of intransitive behavior encountered in several physical or chemical processes. To do so, our main tools are the concept of an interval order and its numerical representability through two real-valued functions, when available. Thus, the main results introduced in Section 5 above can indeed be used to explain typical intransitivities observed in Thermodynamics, when dealing with physical or chemical processes.

Remark 28. However, in Section 6, bearing in mind the results launched in Section 5, we are going to interpret only the case of intransitivities that derive from an interval order. Those intransitivities could be dealt with by means of two different entropy functions. However, when the intransitivity is of a totally different nature, the corresponding study remains open. At this stage, we should notice that, obviously, not all the intransitive binary relations that may be defined on a nonempty set are related to interval orders in some way. There are other different kinds of intransitivity, that we do not consider in the present manuscript.

To start with, let us notice first that the numerical representability of an interval order $\prec$ on a nonempty set $X$, by means of a pair $(u, v)$ of real-valued functions such that $a \prec b \Leftrightarrow v(a)<u(b)$ holds true for every $a, b \in X$ has already been characterized in Theorem 4 , through the condition of interval order separability.

However, nothing has been said yet concerning the possibility of interpreting Theorem 4 in a thermodynamical setting, in terms of entropies. For instance, we may wonder if the functions $u, v$ that appear in a pair that represents the interval order $\prec$ are indeed entropies in some interpretation ad hoc.

A starting point to give a positive answer to the last question is the interpretation of the traces associated to an interval order $\prec$ on a nonempty set $X$. As previously stated in Definition $13, a \prec^{*} b \Leftrightarrow$ $a \prec c \precsim b$ for some $c \in X$, and $a \prec^{* *} b \Leftrightarrow a \precsim c \prec b$ for some $c \in X \quad(a, b \in X)$. A glance at the last expressions shows that $\prec^{*}$ (respectively, $\prec^{* *}$ ) appears as the composition “ $\prec \circ \precsim$ " (respectively,

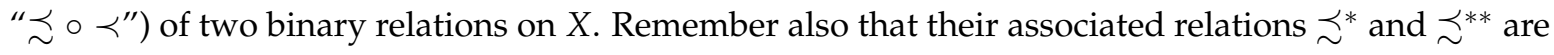
actually total preorders on the given set $X$ (see, e.g., [45]).

Given $a, b \in X$, we may observe that $a \precsim^{*} b \Leftrightarrow \neg\left(b \prec^{*} a\right)$. However, the negation of $b \prec^{*} a$ just states that there is no $c \in X$ such that $b \prec c \precsim a$. In other words, for any $c \in X$ it holds true that $b \prec c \Rightarrow a \prec c$. Calling $R(x)=\{c \in X: x \prec c\} \quad(x \in X)$, we arrive at $a \precsim^{*} b \Leftrightarrow R(b) \subseteq R(a)$. Similarly, we also have that $a \precsim^{* *} b \Leftrightarrow \neg\left(b \prec^{* *} a\right)$, and here the negation of $t \prec^{* *} s$ tells us that there is no $c \in X$ such that $b \precsim c \prec a$. Equivalently, for all $c \in X$ it holds true that $c \prec a \Rightarrow c \prec b$. Hence, calling $L(x)=\{c \in X: c \prec x\} \quad(x \in X)$, we get that $a \precsim^{* *} b \Leftrightarrow L(a) \subseteq L(b)$.

If $\prec$ is representable through a pair $(u, v)$, this kind of representation can still be improved, as stated in the next crucial Theorem 8 that refines Theorem 4 in Section 5.

Theorem 8. Let $X$ be a nonempty set. Let $\prec$ stand for an interval order defined on the given set $X$. The following statements are equivalent:

(i) The interval order $\prec$ is representable through a pair $(u, v)$ of real-valued functions (i.e., $a \prec b \Leftrightarrow v(a)<$ $u(b)$ holds true for every $a, b \in X)$. 
(ii) The interval order $\prec$ is representable through a pair $(U, V)$ of real-valued functions, with the additional property that $U$ represents the total preorder $\precsim^{* *}$ (that is, $a \precsim * * b \Leftrightarrow U(a) \leq U(b)(a, b \in X)$ ), while $V$ represents the total preorder $\precsim *$ (i.e., $a \precsim * b \Leftrightarrow V(a) \leq V(b)$ holds true for every $a, b \in X$ ).

Proof. See [45], Theorem 1 on pp. 175-181.

Remark 29. This Theorem 8 is not stating that any numerical representation $(u, v)$ of $\prec$ is such that $u$ represents $\precsim^{* *}$ and $v$ represents $\precsim^{*}$. Instead, it is said is that if there is a pair $(u, v)$ that represents the interval order $\prec$, then there is another one $(U, V)$ (perhaps different!) with $U$ representing $\precsim * *$ and $V$ representing $\precsim *$. By the way, notice that, unlike Theorem 6, Theorem 8 does not involve continuity.

Going back to the thermodynamical approach, let us now discuss how this last result, namely Theorem 8, can actually be conciliated in a way with Carathéodory's axiomatics, in order to study situations when the accessibility relation on the state space $S$ is intransitive. As already implicitly suggested in [73], to interpret certain processes, perhaps more than one entropy should be considered.

In Klimenko's own words (see [73]):

$<<$ In non-equilibrium phenomena, the production of physical entropy is typically high, in perfect argument with the laws of thermodynamic. Although no direct violation of the laws of thermodynamics is known, thermodynamics struggles to explain complexity, which is often observed in essentially non-equilibrium phenomena: turbulent mixing and combustion as well as evolution of life forms may serve as typical examples. The entropy of turbulent fluctuations does not seem to be maximal and the same applies to entropies characterizing distributions in other complex non-equilibrium processes. These entropies have similarities with but are not the same as the molecular entropy, which characterizes disorder of molecular movements and is subject to the laws of thermodynamics. We use the term apparent entropy to distinguish entropy-like quantities from the molecular entropy. $>>$

This key idea, namely the use of more than one entropy seems to support also the observation made in [38], already mentioned before, namely:

$<<$ Physical thermodynamics is always transitive, but the same cannot be stated a priori about apparent thermodynamics. $>>$

In other words, it seems that in several processes at least two (instead of just one) different kinds of thermodynamical behavior, that we may call "physical" and "apparent" are involved. Thus, it seems adequate to consider, too, two different but closely related kinds of entropy instead of just one. In our opinion, hopefully this could help a physicist or a chemist to interpret and understand better practical situations where some kind of intransitivity is observed.

In the study made in [73], concerning thermodynamical behavior of intransitive competition, it is said that competition is intransitive if at least one intransitive triplet arises.

Klimenko [73] then claims that:

$<<$ Generally, a consistent ranking cannot be introduced for intransitive competition, but ranking can often be assigned to subdomains if the competition is transitive within these subdomains. It should be noted, however, that the rankings assigned in different subdomains would result in multi-valued functions and can not be made fully consistent with each other when the competition is intransitive. $>>$

Unlike Klimenko's approach in [73], we should point out here that Theorem 8 carries the following important suggestions:

(i) Instead of just one ranking, we would need to use two. One of them is given by $U$ and the other one comes from by $V$.

(ii) Both functions $U, V$ act on the whole domain. It is unnecessary to consider here subdomains of transitivity of the interval order given a priori on the set $X$.

(iii) The functions $U$ and $V$ can be understood as "fully consistent" one another. They are interdependent because they represent, jointly, the given interval order. 
Coming back to the thermodynamical setting once more, now we may suppose that the state space $S$ is equipped with an accessibility relation (asymmetric in this case), say $\hookrightarrow$, that is now an interval order. We may now consider its traces and their corresponding adjoints. Remember that those adjoints are total preorders on the phase system $S$. This would allow us to use the Carathéodory axioms independently for each of the adjoints of the traces of $\hookrightarrow$ in order to find entropy functions that represent them, asumming that the condition of perfect separability, necessary to warrant the existence of a utility function that represents a total preorder, as stated in Theorem 1, is accomplished by both such adjoints. Thus, this approach is consistent with the set of axioms due to Carathéodory and Cooper (see [18]), the classical one in Thermodynamics.

However, using the theory on interval orders we can actually go further. Theorem 8 actually allows us to construct both entropy functions $U, V$ at the same time, and with the additional conditions that the pair $(U, V)$ represents $\hookrightarrow$ and, in addition, $U(s) \leq V(s)$ holds for every $s \in S$ so that $U$ and $V$ could indeed be interpreted as a pair of entropies such that one of them dominates (i.e., is bigger than) the other, as in the typical case of apparent entropy vs. molecular entropy encountered in physical and chemical processes.

Hence, to deal with intransitivities in a reasonable way by means of an intertwined pair of entropy functions, we need that the state space $S$ endowed with the binary relation $\hookrightarrow$ be an interval order that satisfies the separability condition. Finally, if we are also interested in continuous representations for $\hookrightarrow$ with respect to some topology $\tau$ on the phase system $S$, a reasonably good restriction on the topology $\tau$ is that $\tau$ be natural as regards the interval order $\hookrightarrow$. In that case Theorem 6 is of capital importance in this setting.

Therefore, following [1] and summarizing all the ideas presented in Section 6, we point out to the physicist or chemist the following suggestions in order to analyze a process in which some intransitivity could be noticed:

(i) If a chemical or physical process shows intransitivities, two entropy functions instead of just one should be used.

(ii) One of these two entropy functions should be smaller than the other one.

(iii) Once both entropy functions have been obtained and identified (this depends on the process considered), try to interpret the intransitivity observed by means of of a representable interval order that models the accessibility relation.

(iv) Other features that could occur aside the intransitivity observed in a chemical or physical process may depend on other different additional mathematical structure. Thus, to analyze questions related to mixing and competition the consideration of some algebraic structure (e.g., a semigroup) could be necessary. In addition, to handle continuity, connectedness or metrizability among other possible features, the use of some topological notions is compulsory.

(v) It is also crucial to identify the mathematical nature of the process to be analyzed. Whereas the mere intransitivity has to do with mathematical ordered structures, mixing and competition force the intervention of algebraic structures, and continuity needs some topology being defined on the state space. Of course, all these mathematical structures do not work independently from each other: they interact in the processes considered, but in a way that each of them, separately, is mainly responsible for some particular aspect of our interest (e.g., intransitivity).

\section{The Construction of Numerical Representations of Ordered Structures}

A further glance at Section 2 with all the preliminaries related to entropy reminds us again that most of the usual definitions of entropy that arise in the frame of the laws of Thermodynamics are given through some integrals.

We could say that this fact comes, and it is justified too, from physical evidence, experiments, etc. Therefore, the mathematical background on entropies acts only as a basis (that maybe we can consider 
a cornerstone), to justify in an abstract and rigorous manner all what, say, a physicist or a chemist is observing in her/his laboratory.

Well, even if all this is formally correct, we may still wonder about the possibility of constructing numerical representations of orderings that are got in terms of some mathematical integral.

To fix ideas, we could start by considering a representable total preorder $\precsim$ defined on a nonempty set $X$. Thus, we know that $\precsim$ admits a utility function that represents it. In fact, if $X$ is endowed with a topology $\tau$ that is natural with respect to $\tau$ it is also possible to find a numerical representation of $\precsim$ that is continuous with respect to the topology $\tau$ on $X$ and the usual Euclidean topology on the real line.

However, now the question is:

Can some numerical representation of $\precsim$ be built in a natural way through some kind of integral? What about continuous utility functions for $\precsim$ ?

This is far from easy!

Here, we should pay attention to the constructions of numerical representations for total preorders that have already been introduced in the literature (e.g., Utility Theory, Entropy, etc.).

Concerning numerical representations of total preorders, some classical constructions appear in [106] and also in the first four chapters of Reference [44], among many other sources.

Thus, for the particular case in which the nonempty set $X$ is finite, and it is endowed with a total (linear) order $\preceq$, given $x \in X$ we may define $n(x)$ as the cardinality of the set $\{z \in X: x \preceq z\}$. It is then clear that $x \preceq y \Leftrightarrow n(x) \leq n(y)$ holds true for any $x, y \in X$. This construction is very old indeed. It essentially appears in Cantor's famous papers [132,133]. George Cantor is considered as the pioneer of the search for numerical representation of ordered structures.

Remark 30. When working with numerical representations of different classical of orderings (e.g., total preorders, interval orders and semiorders) defined on finite sets, the mathematical techniques mainly come from Combinatorics (an excellent book in this direction is [134]). However, when working with infinite sets, the techniques are quite different, and are based on General Topology, Real Analysis, etc. An excellent book in this setting is [44].

Now, suppose that $X$ is infinite but countable, say $X=\left\{x_{n}: n \in \mathbb{N}\right\}$ and it is endowed with a total order $\prec$. In this case, we define a suitable function $u: X \rightarrow \mathbb{R}$ as follows:

$$
u\left(x_{k}\right)=\sum_{i: x_{i} \preceq x_{k}} \frac{1}{2^{i}} \quad(k \in \mathbb{N}) .
$$

Again, it is straightforward to see that $x_{k} \preceq x_{l} \Leftrightarrow u\left(x_{k}\right) \leq u\left(x_{l}\right) \quad(k, l \in \mathbb{N})$, so $u$ is a utility function that represents $\preceq$.

When $X$ is infinite (but maybe it is uncountable now), and $\preceq$ is a perfectly separable total order on $X$, first we take a countable subset $D \subseteq X$ such that given $a, b \in X$ with $a \prec b$ there exists $d \in D$ such that $a \preceq d \preceq b$ (see Theorem 1 in Section 3). Then, we represent the restriction of $\preceq$ to the countable subset $D$ by means of a suitable series, as before. Finally, a utility function $u$ defined on $D$ can be extended to the whole set $X$. To do so, given $x \in X$ we may define $u(x)=\sup \{u(d): d \preceq x, d \in D\}$. We may prove that this extension defines a numerical representation for $\preceq$ on the set $X$.

From the point of view of continuity, provided that $X$ is endowed with some topology $\tau$ that is natural with respect to the total order $X$, all the above constructions, based on series, are troublesome. The reason is that they give rise to discontinuities at each $d \in D$. This is really a pity, because it forces us to use other different techniques if we want to have at hand continuous utility functions. At this stage, the famous Debreu's open gap lemma (see Lemma 1 in Section 3) comes to our rescue. Through that key result, we can finally build a continuous utility function representing $\preceq$. However, almost all the known proofs of Lemma 1 are quite difficult, and unintuitive in the sense that the construction is too theoretical and far beyond a possible visualization of it. 
Anyway, by means of Debreu's open gap lemma we warrant the existence of continuous utility functions. However, this does not solve our former question yet, since we do not know whether or not such new continuous utility function appears as a suitable integral.

Again, a new step is necessary. Thus, if $u: X \rightarrow \mathbb{R}$ is a utility function that represents $\preceq$ and is continuous with respect to the order topology $\tau_{\preceq}$ on $X$ and the usual topology on the real line, by composing $u$ with another suitable continuous an strictly increasing function we may consider without loss of generality that $u$ takes values in $(0,1)$. Then, we can "transport" the Lebesgue measure $\mu$ on the unit interval $(0,1)$, to the set $X$, as follows: By definition, now a measurable set of $X$ is a subset $Y \subseteq X$ such that $\{u(y): y \in Y\} \subseteq(0,1)$ is Lebesgue-measurable. In this way, we could interpret $\mu(\{u(y): y \in Y\})$ as the "transported" measure of $Y$. In this way, the Lebesgue integral on $(0,1)$ could also be transported to $X$ by means of the utility function $u$.

As can be seen, all this needs too many steps, and our intuition could be lost in the process. However, at least, we may say that it is theoretically possible to have at hand continuous ad hoc numerical representations of total orders that are defined by means of some integral.

To conclude Section 7, we point out that in the specialized literature about numerical representations of ordered structures, the use of integrals to define utility functions is quite scarce. (See e.g., $[44,64,65,67,90])$.

\section{Discussion}

As analyzed in Section 6, intransitivities encountered in various physical or chemical processes could be handled by means of interval orders, and a pair of entropies instead of just one.

We already know that the classical numerical representations of interval orders that are interval order separable use two real-valued functions. However, we may say that this is because, given a nonempty set $X$ endowed with an interval order $\prec$, we want to represent $\prec$ by means of real-valued maps. At this point, we may think of other alternative representations in which the codomain of the functions used is no longer the real line $\mathbb{R}$, but, instead, some other suitable set, say $Z$, also endowed with some ordering ad hoc. In this direction, in some papers in the specialized literature, other codomains have already been used, e.g., nonstandard numbers (see, e.g., [135]), sets of fuzzy numbers (see [136]), suitable subsets of the unit square or similar (see $[120,137,138]$ ), or lexicographic products and powers (see $[139,140])$. The idea is that the codomain be so that in order to represent, say, total preorders, interval orders or semiorders only one mapping from $X$ into $Z$ (see also [141]).

At this point, a remarkable fact is that this setting, that apart from pure mathematics has also been used, for instance, in Utility Theory in Economics, has not been implemented yet in Thermodynamics, when studying intransitivities and their corresponding interpretations by means of entropies. This setting would then give rise to consider entropy functions that, instead of taking values on the real line, should be defined in a way such that their codomains are alternative sets with good properties. As far as we know, this would be new, and maybe it should also generate some rewriting of the laws of Thermodynamics in other quite different terms, to give room, say, to entropy functions defined by integrals that do not take values on the real line but on other alternative measure spaces that should also be endowed with some total order ad hoc.

\section{Further Comments, Open Problems and Conclusions}

\subsection{Some Further Comments}

Our intention in this paper has been to present a survey on the main results arising in the theory of numerical representations of ordered structures. This is the mathematical background that helps us to understand better the notion of an entropy arising in Thermodynamics.

At least, this mathematical approach highlights the axiomatic setting to the concept of Entropy, issued in Carathéodory's pioneer papers (see [51]). However, it is also important to say that not only Entropy, but other interdisciplinary settings, lean on exactly the same mathematical background. This 
is the case of Utility Theory in Economics (see [2-9]), Information Theory (see [14]), Measurement Theory in Psychology (see, e.g., [12]), or even Relational Systems arising in First Order Logic (see [142]). Therefore, it seems necessary to pay attention to the fact that some results arising, say, in Utility Theory can also be seen in Entropy Theory in Thermodynamics, of course using a different language and nomenclature, and adapted to the corresponding setting.Vice versa, some results arising in axiomatic Thermodynamics are indeed equivalent to classical results in Utility Theory, Measurement Theory, etc. We are not saying here that, say, a physicist interested in entropies should be acquainted with results arising in Economic or Psychology. What we want to point out is that all of those scientific collectives (physicists, chemists, economists, theoretical psychologists, etc.) should be aware of the fact that theories they are dealing with have exactly the same basis. Therefore, all of them should be used to working someway in a more abstract pure mathematical setting, that is in fact the cornerstone and root of the theory they are studying, no matter which it be. To put an evident example, we may consider again the Debreu's open gap lemma, now recognized in Mathematics as one of the most powerful results in the theory of ordered structures. That result was launched through a journal devoted to Economics, namely the "International Economic Review".

At this stage, physicists could believe that, to put only an example, Utility Theory in Economics could actually lead to the construction of suitable entropy functions in, say, Thermodynamics. Conversely, an economist could think that the knowledge of Thermodynamics could help her/him to build utility functions. However, what really happens is that both theories share the same mathematical basis. Therefore, if we go down to that ground level and look at the abstract results, we can then use them in our particular field of interest, no matter if it comes from Economics, Physics or other disciplines. From Utility Theory to Entropy, the path to do so is the following: (1) Consider a classical result in Utility Theory. (2) Put it in mathematical terms, as a pure abstract result arising in the theory of numerical representation of orderings. (3) Rewrite it in physical terms, so that, for instance, the space considered is a phase system, and the numerical representation is an entropy function.

Let us analyze this through an easy example, in which the corresponding interpretation would depend on the discipline we are working in.

Example 3. Consider the following graph:

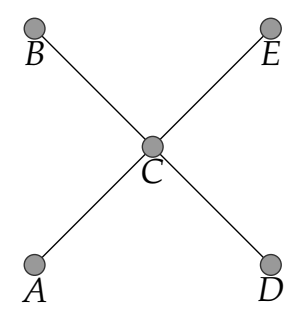

1. For a mathematician, this graph could be interpreted as a total preorder $\precsim$ on the set $X=\{A, B, C, D, E\}$ such that $A \sim A, A \sim B, A \prec C, A \prec D, A \prec E, B \sim B, B \prec C, B \prec D, B \prec E, C \sim C, C \prec D, C \prec$ $E, D \sim D, D \sim E, E \sim E$. A numerical representation for this total preorder is the function $u: X \rightarrow \mathbb{R}$ given by $u(A)=u(B)=1 ; u(C)=2, u(D)=u(E)=3$.

2. For people working in Computer Science, this graph could represent tasks to be done for several computers that are connected between them. The task represented by the node $C$ needs that the tasks $A$ and $B$ be finished. In addition, before starting the tasks represented by the nodes $D$ and $E$, we need that the task represented by $C$ be concluded. Hence, maybe using a topological sorting (see [34]) or any sort of graph entropy (see [32]), we would decide how to start and distribute the tasks (e.g: $A-B-C-D-E$, $B-A-C-E-D$, etc.).

3. For a chemist, this graph could represent a reaction, such as $\mathrm{HCl}+\mathrm{NaOH} \rightarrow \mathrm{NaCl}+\mathrm{H}_{2} \mathrm{O}$. Node A (respectively, $B$ ) represents a recipient with $\mathrm{HCl}$ (respectively, with $\mathrm{NaOH}$ ), node $\mathrm{C}$ is another recipient where these components react, and nodes $D$ and E correspond to new recipients that collect the results of 
the reaction, namely salt and water. From the point of view of entropy, we could understand this as a phase system $\mathcal{S}=\{s, t, u\}$ such that $s=\{A, B\}, t=\{C\}$ and $u=\{D, E$,$\} such that s \rightsquigarrow^{*} t \rightsquigarrow^{*} u$. This means that the process of taking acid and base, letting them react, and getting salt and water is irreversible. In terms of some entropy $f: \mathcal{S} \rightarrow \mathbb{R}$ we should have that $f(s)<f(t)<f(u)$.

4. For people working in Psychology and trying to measure human skills, the graph could represent a situation of a person that studies two disciplines (e.g., Linguistic and Poetry) represented by nodes $A$ and $B$, obtains a Ph.D. represented by node $C$ and then publishes two books represented by nodes $C$ and $D$. This can also be controlled by a measurement function, say $m:\{A, B, C, D, E\} \rightarrow \mathbb{R}$ given by $m(A)=m(B)=1 ; m(C)=2, m(D)=m(E)=3$, that gives an idea of the evolution of the skills of such person, so that $\{A, B\}$ (first level) correspond to studying, $\{C\}$ (second level) corresponds to obtaining $a$ Ph.D, and $\{C, D\}$ (third level) correspond to having got the ability of publishing books.

5. For an economist the previous graph could correspond to a situation in which a firm wants to hire people among five candidates $\{A, B, C, D, E\}$ such that $A$ and $B$ are equally good, whereas $C$ is better than $A$ or $B$, and finally $D$ is equally good to $E$ and better than $C$. This can also be represented by a suitable utility function $U:\{A, B, C, D, E\} \rightarrow \mathbb{R}$, for instance, $U(A)=U(B)=8 ; U(C)=9, U(D)=U(E)=10$ counting the points obtained for each candidate in a test consisting of ten items.

To this extent, perhaps it is necessary to have at hand a good mathematical journal that could collect the new advances obtained on those theoretical and abstract setting, so that all multidisciplinary scientific collectives could use it as a main reference for abstract results. Well, this was the idea of the foundation of the journal "Order" (to put only a clear example). However, that journal was launched in 1984, and, obviously, most classical results arising in Entropy Theory, Utility Theory, Measurement Theory, Mathematical Theory of Ordered Structures, etc., had already appeared much earlier, and, unfortunately, are dispersed in a wide sort of scientific journals.

In fact, many classical and crucial results in the mathematical theory of numerical representations of ordered structures arise for the first time in journals that were perhaps less well known to the pure mathematician researching, say, in Algebra or Differential Calculus.

Thus, even if they are commonly recognized by mathematicians as some of the key concepts and results in the theory of numerical representations of ordered structures, and consequently incorporated to the mathematical texts in this theory, some crucial achievements were published in journals devoted to Economics or Psychology. This is the case of the famous Debreu's open gap lemma (see [54]) or the study of the main properties of interval orders (see [42]). Indeed, there is a strong tradition of working with ordered structures in journals such as the "Journal of Mathematical Economics" or the "Journal of Mathematical Psychology".

From the point of view of the pure mathematician that works with ordered structures, it is a pity to realize that the topic "Order" is dispersed in various mathematical disciplines including Algebra, Mathematical Analysis, Combinatorics or General Topology. Unfortunately, it seems not to have yet a sort of status that could locate it at the same level as the other classical branches of Mathematics.

Recently, the journal "Axioms" pays attention to constructions based on an axiomatic setting. Obviously, some of those constructions could be related to ordered structures and their interdisciplinary applications, as shown in the present manuscript.

\subsection{Open Problems and Suggestions for Further Research}

Among open problems arising in this theory, already in its mathematical background, we point out the following ones:

(i) A general characterization of the continuous representability of interval orders has not been achieved up-to-date.

(ii) No general characterization of the existence of a continuous Scott-Suppes representation of a semiorder is known yet, either. 
(iii) A directconstruction of numerical representations of total preorders based on some integral is also unknown.

In what concerns entropies, an open problem could be the consideration of the concept of entropy through integrals that take values in codomains different from the real line. Perhaps this could force to rewrite some aspects of the laws of Thermodynamics.

We furnish now some appealing suggestion for further research that is based in the notion of a Scott-Suppes representable semiorder, already considered in Section 5. We remind the reader that Definition 15 states that a semiorder $\prec$ defined on a nonempty set $X$ is representable in the sense of Scott and Suppes if there exists a function $u: X \rightarrow \mathbb{R}$ and a constant $k>0$ such that $a \prec b \Leftrightarrow u(a)+k<u(b)$ holds true for every $a, b \in X$. What is really important here is that such constant $k>0$ can be interpreted as a "threshold of discrimination" (see, e.g., $[39,40]$ ), or maybe as a "quantum". Thus, through the function $u$, two objects $x, y \in X$ that differ in less than a "quantum" $k$ (i.e., $|u(x)-u(y)|<k$ ) can be, in a sense, considered indifferent. In some applications that may arise in Physics, Chemistry, etc., perhaps this could lead us to consider two events as "indistinguishable". In this direction, in Chemistry and Physics nowadays one may encounter situations that have a well-deserved growing interest (e.g., small scales, multiscale analysis, see $[143,144])$ and could give room to the use of the notion of a "quantum". The use of semiorders to analyze some particular features of quantum theories has already been considered in the corresponding literature (see, e.g., $[145,146])$. Nevertheless, its methodical and standard use as a potentially helpful device to handle quantum processes arising in Chemistry and/or Physics seems to remain still open. It is important to point out at this stage that the concept of a "quantum" has already been used in Entropy Theory or, more generally, in Thermodynamics (see, e.g., [147-150]). Therefore, the theory of Scott-Suppes representable semiorders could play an important role in next future to understand better situations that, related to some intransitivities, arise in various quantum theories.

\subsection{Concluding Remarks}

Following the ideas already launched in [1], we can declare here that our aim has been to show facts and results which can be framed and interpreted by means of non-routine mathematical methodologies often unfamiliar to the usual audience of mainstream experimental and theoretical Chemistry and/or Physics journals, and in particular those devoted to the study of Entropy. To do so, we have presented here the main results arising in the mathematical theory of the numerical representations of ordered structures, trying to put in evidence that this is the mathematical background on which Entropy Theory leans. Therefore, we have tried to explain how several key concepts arising in the axiomatics encountered in Entropy Theory correspond to items that are classical topics in the mathematical theory of ordered structures. In particular, the use of interval orders and semiorders to interpret intransitivities is a key point to reach our objective.

Our set and exposition of ideas has been abstract, with plenty of mathematical formalism. It was not our intention to analyze some concrete example of a chemical or physical intransitive process. Instead, we wanted to furnish a new mathematical basis to set well and interpret those situations, and how can we handle them using classical ideas coming from Thermodynamics, and, in particular Carathéodory axioms, as well as its mathematical background consisting of interval order and semiorder theory. The analysis of practical situations encountered in processes and experiments is, consequently, left open. However, we recommend the reader interested in concrete applications and/or practical constructions to have a look to several of the sources that appear in the list of references. To put only an example, we point out that in [38] a wide variety of real life situations where intransitivities appear have been considered and analyzed in depth. In addition, it is interesting to have a look to the references $[1,2,5-7,9,10,12-14,16,17]$ to compare many interdisciplinary uses of entropy and related items, where several practical situations and examples in each discipline have also been considered and discussed. 
Author Contributions: Two of the authors of this paper, namely M.-J.C. and E.I., are mathematicians. One of us (A.R.-P.) has degrees in both Mathematics and Physics. C.G.-P. is a physicist. Obviously, the mathematicians have paid more attention to all that has to do with numerical representability of ordered structures, whereas the physicists have put the emphasis in checking all that has to do with entropies. In addition, M.-J.C. and A.R.-P. are currently working in an institute devoted to the study of topics related to Economics, so that all the mentions to utility theory throughout the manuscript have also been controlled and checked by them.

Acknowledgments: This work was partially supported by the research projects ECO2015-65031-R, MTM2015-63608-P (MINECO/AEI-FEDER, UE), 0011-1365-2016-000060, PC003-004-005 DRUG-MAG, TIN2016-77356-P (MINECO/AEI-FEDER, UE) and 0011-1365-2017-000118. Thanks are given to the editors and two anonymous referees for their valuable suggestions and comments that have led to many improvements included in this version of the manuscript.

Conflicts of Interest: The authors declare no conflict of interest.

\section{References}

1. Campión, M.J.; Arzamendi, G.; Gandía, L.M.; Induráin, E. Entropy of chemical processes versus numerical representability of orderings. J. Math. Chem. 2016, 54, 503-526.

2. Candeal, J.C.; De Miguel, J.R.; Induráin, E.; Mehta, G.B. Utility and entropy. Econom. Theory 2001, 17, $233-238$.

3. Candeal, J.C.; De Miguel, J.R.; Induráin, E.; Mehta, G.B. On a theorem of Cooper. J. Math. Anal. Appl. 2001, $258,701-710$.

4. Candeal, J.C.; De Miguel, J.R.; Induráin, E.; Mehta, G.B. Representations of ordered semigroups and the physical concept of entropy. Appl. Gen. Topol. 2004, 5, 11-23.

5. Sousa, T.; Domingos, T. Is neoclassical microeconomcs formally valid? An approach based on an analogy with equilibrium thermodynamics. Ecol. Econ. 2006, 58, 160-169.

6. Sousa, T.; Domingos, T. Equilibrium econophysics: A unified formalism for neoclassical economics and equilibrium thermodynamics. Phys. A 2006, 371, 492-612.

7. Bryant, J. A thermodynamic theory of economics. Int. J. Exergy 2007, 4, 302-337.

8. Smith, E.; Foley, D.K. Classical thermodynamics and economic general equilibrium. J. Econom. Dyn. Control 2008, 32, 7-65.

9. Dionisio, A.; Reis, A.H.; Coelho, L. Utility function estimation: The entropy approach. Physicial A 2008, 387, 3862-3867.

10. Shubik, M.; Smith, E. Building theories of economic process. Complexity 2009, 14, 77-92.

11. Pokrovskii, V.N. Econodynamics: The Theory of Social Production. In New Economic Windows; Springer: Dordrecht, The Netherlands, 2012.

12. Roberts, F.S.; Luce, R.D. Axiomatic thermodynamics and extensive measurement. Synthese 1968, 18, 311-326.

13. Davis, P.J. Entropy and Society: Can the physical/mathematical notions of Entropy be usefully imported into the social sphere? J. Hum. Math. 2011, 1, 119-136.

14. Shannon, C.E. A mathematical theory of communication. Bell Syst. Tech. J. 1948, 27, 379-423.

15. Meucci, A. Fully flexible views: Theory and practice. Risk 2008, 21, 97-102.

16. Zhou, R.; Cai, R.; Tong, G. Applications of Entropy in Finance: A review. Entropy 2013, 15, 4909-4931.

17. Kosko, B. Fuzzy entropy and conditioning. Inf. Sci. 1986, 40, 165-174.

18. Cooper, J.L.B. The foundations of Thermodynamics. J. Math. Anal. Appl. 1967, 17, 172-193.

19. Lieb E.H.; Yngvason, J. The Physics and Mathematics of the second law of Thermodynamics. Phys. Rep. 1999, 310, 1-96.

20. Landsberg, P.T. Main ideas in the axiomatics of thermodynamics. Pure Appl. Chem. 1970, 22, $215-228$.

21. Lieb, E.H.; Yngvason, J. A guide to entropy and the second law of thermodinamics. N. Am. Math. Soc. 1998, 45, 571-581.

22. Pogliani, L.; Berberan-Santos, M.N. Constantin Carathéodory and the axiomatic thermodynamics. J. Math. Chem. 2000, 28, 313-324.

23. Zachmanoglou, E.C. Carathéodory theorem on the second law of thermodynamics. SIAM J. Appl. Math. 1973, 25, 592-596.

24. Mosterín, J. Conceptos y teorías en la ciencia; Alianza Editorial: Madrid, Spain, 1984.

25. Gibbs, J.W. Elementary Principles in Statistical Mechanics; Charles Scribner's Sons: New York, NY, USA, 1902.

26. Von Neumann, J. Mathematische Grundlagen der Quantenmechanik; Springer: Berlin, Germany, 1932. 
27. Jaynes, E.T. Information theory and statistical mechanics. Phys. Rev. 1957, 106, 620-630.

28. Knuth, K.H. Deriving laws from ordering relations. AIP Conf. Proc. 2004, 707, 204-235.

29. Knuth, K.H.; Skilling, J. Foundations of inference. Axioms 2012, 1, 38-73.

30. Maluf, J.W. The teleparallel equivalence of general relativity and the gravitational centre of mass. Universe 2016, 2, 19.

31. Dugundji, J. Topology; Allyn and Bacon: Boston, MA, USA, 1966.

32. Dehmer, M. Information processing in complex networks: Graph entropy and information functionals. Appl. Math. Comput. 2008, 201, 82-94.

33. Kahn, A.B. Topological sorting of large networks. Commun. ACM 1962, 5, 558-562.

34. Knuth, D.E.; Szwarcfiter, J.L. A structured program to generate all topological sorting arrangements. Inf. Process. Lett. 1974, 2, 153-157.

35. Debreu, G. Representations of a preference ordering by a numerical function. In Decision Processes; Thrall, R., Coombs, C., Davies, R., Eds.; John Wiley: New York, NY, USA, 1954.

36. Hicks, J. A Revision of Demand Theory; Clarendon Press: Oxford, UK, 1956.

37. Debreu, G. Theory of Value; John Wiley: New York, NY, USA, 1959.

38. Klimenko, A.Y. Intransitivity in Theory and in the Real World. Entropy 2015, 17, 4364-4412.

39. Luce, R.D. Semiorders and a theory of utility discrimination. Econometrica 1956, 24, 178-191.

40. Scott, D.; Suppes, P. Foundational aspects of theories of measurement. J. Symb. Log. 1958, 23, $113-128$.

41. Wiener, N. Contribution to the theory of relative position. Math. Proc. Camb. Philos. Soc. 1914, 17, 441-449.

42. Fishburn, P.C. Intransitive indifference with unequal indifference intervals. J. Math. Psychol. 1970, 7, 144-149.

43. Fishburn, P.C. Intransitive indifference in preference theory: A survey. Oper. Res. 1970, 18, 207-228.

44. Bridges, D.S.; Mehta, G.B. Representations of Preference Orderings; Springer: Berlin/Heidelberg, Germany; New York, NY, USA, 1995.

45. Bosi, G.; Candeal, J.C.; Induráin, E.; Olóriz, E.; Zudaire, M. Numerical representations of interval orders. Order 2001, 18, 171-190.

46. Bosi, G.; Campión, M.J.; Candeal, J.C.; Induráin, E. Interval-valued representability of qualitative data: The continuous case. Int. J. Uncertain. Fuzziness Knowl. Based Syst. 2007, 15, $299-319$.

47. Van Ness, H.C. Understanding Thermodynamics; Dover: London, UK, 1969.

48. Clausius, R. Über die Wärmeleitung gasförmiger Körper. Ann. Phys. 1865, 125, 353-400.

49. Pastor, J.M.; Pérez-Landazábal, J.I.; Gómez-Polo, C.; Recarte, V.; Larumbe, S.; Santamarta, R.; Fernandes Silva, M.; Gómez Pineda, E.A.; Winkler Hechenleitner, A.A.; Lima, M.K. Entropy change linked to the magnetic field induced Morin transition in Hematite nanoparticles. Appl. Phys. Lett. 2012, 100, 063102.

50. Born, M. National Philosophy of Cause and Chance; Oxford: London, UK, 1949.

51. Carathéodory, C. Untersuchungüber die Grundlagen der Thermodynamik. Math. Ann. 1909, 67, 355-386.

52. Sánchez-Alarcos, V.; Recarte, V.; Pérez-Landazábal, J.I.; Gómez-Polo, C.; Chernenko, V.A.; González, M.A. Reversible and irreversible martensitic transformations in Fe-Pd and Fe-Pd-Co alloys. Eur. Phys. J. Spec. Top. 2008, 158, 107-112.

53. Eilenberg, S. Ordered topological spaces. Am. J. Math. 1943, 63 39-45.

54. Debreu, G. Continuity properties of Paretian utility. Int. Econom. Rev. 1964, 5, 285-293.

55. Ciesielski, K. Set Theory for the Working Mathematician; Cambridge University Press: Cambridge, UK, 1997.

56. Campión, M.J.; Candeal, J.C.; Induráin, E.; Mehta, G.B. Continuous order representability properties of topological spaces and algebraic structures. J. Korean Math. Soc. 2012, 49, 449-473.

57. Jaffray, J.-Y. Existence of a continuous utility function: An elementary proof. Econometrica 1975, 43, 981-983.

58. Herden, G. On the existence of utility functions. Math. Soc. Sci. 1989, 17, 297-313.

59. Candeal, J.C.; Induráin, E. Lexicographic behaviour of chains. Arch. Math. 1999, 72, 145-152.

60. Beardon, A.F.; Candeal, J.C.; Herden, G.; Induráin, E.; Mehta, G.B. The non-existence of a utility function and the structure of non-representable preference relations. J. Math. Econom. 2002, 37, 17-38.

61. Steen, L.A.; Seebach, J.A., Jr. Counterexamples in Topology; Dover: New York, NY, USA, 1995.

62. Rader, T. The existence of a utility function to represent preferences. Rev. Econ. Stud. 1963, 30, $229-232$.

63. Mehta, G.B. The Euclidean distance approach to continuous utility functions. Quart. J. Econom. 1991, 106, 975-977.

64. Candeal-Haro, J.C.; Induráin-Eraso, E. Utility representations from the concept of measure. Math. Soc. Sci. $1993,26,51-62$. 
65. Candeal-Haro, J.C.; Induráin-Eraso, E. Utility representations from the concept of measure: A corrigendum. Math. Soc. Sci. 1994, 28, 67-69.

66. Mehta, G.B. A remark on a utility representation theorem of Rader. Econom. Theory 1997, 9, 367-370.

67. Bowen, R. A new proof of a theorem in utility theory. Int. Econom. Rev. 1968, 9, 374.

68. Droste, M. Ordinal scales in the theory of measurement. J. Math. Psychol. 1987 31, 60-82.

69. Beardon, A.F. Debreu's gap theorem. Econom. Theory 1992, 2, 150-152.

70. Herden, G.; Mehta, G.B. Open gaps, metrization and utility. Econom. Theory 1996, 7, 541-546.

71. Bosi, G.; Mehta, G.B. Existence of a semicontinuous or continuous utility function: A unified approach and an elementary proof. J. Math. Econom. 2002 38, 311-328.

72. Alcantud, J.C.R.; Bosi, G.; Campión, M.J.; Candeal, J.C.; Induráin, E.; Rodríguez-Palmero, C. Continuous utility functions through scales. Theory Decis. 2008, 64, 479-494.

73. Klimenko, A.Y. Mixing, entropy and competition. Phys. Scr. 2012, 85, 068201.

74. Klimenko, A.Y. Complex competitive systems and competitive thermodynamics. Philos. Trans. R. Soc. Lond. Ser. A Math. Phys. Eng. Sci 2013, 271, 20120244.

75. Klimenko, A.Y. What is mixing and can it be complex? Phys. Scr. 2013, 2013, T155.

76. Klimenko, A.Y. Complexity and intransitivity in technological development. J. Syst. Sci. Syst. Eng. 2014, 23, $128-152$.

77. Klimenko, A.Y. Entropy and equilibria in competitive systems. Entropy 2014, 16, 1-22.

78. Duda, J.; Milewska-Duda, J. A theoretical model for evaluation of configurational entropy of mixing with respect to shape and size of particles. J. Math. Chem. 1995, 17, 69-109.

79. Gibbs, J.W. On the equilibrium of heterogeneous substances. In Collected Works of J. Willard Gibbs; Longman: New York, NY, USA, 1928; Volume 1, pp. 55-349.

80. Guggenheim, E.A. Modern Thermodynamics by the Methods of Willard Gibbs; Methuen: London, UK, 1933.

81. Candeal, J.C.; Induráin, E.; Sanchis, M. Order representability in groups and vector spaces. Expo. Math. 2012, 30, 103-123.

82. Hölder, O. Der Axiome der Quantität und die Lehre vom Mass. Berichte über die Verhandlungen der Königlich Sachsischen Gesellschaft der Wissenschaften zu Leipzig Math. Phys. Kl. 1901, 53, 1-64.

83. Iseki, K. On simple ordered groups. Port. Math. 1951, 10, 85-88.

84. Chehata, C.G. On an ordered semigroup. Proc. Lond. Math. Soc. 1953, 28, 353-356.

85. Krull, W. Über die Endomorphismen von total geordneten archimedischen abelschen Gruppen. Math. Z. 1960, 74, 81-90.

86. Falmagne, J.C. A set of independent axioms for positive Hölder systems. Philos. Sci. 1975, 42, 137-151.

87. Nyikos, P.J.; Reichel, H.C. Topologically orderable groups. Gen. Topol. Appl. 1975, 5, 195-204.

88. Holland, W.; Smith, D.P. Irrational ordered groups. Order 1988 4, 381-386.

89. Fuhrken, G.; Richter M.K. Additive utility. Econom. Theory 1991 1, 83-105.

90. Candeal-Haro, J.C.; Induráin-Eraso, E. Utility functions on partially ordered topological groups. Proc. Am. Math. Soc. 1992, 113, 765-767.

91. Glass, A.M.W. Partially Ordered Groups; World Scientific: Singapore, 1999.

92. Point, F. Essentially periodic ordered groups. Ann. Pure Appl. Log. 2000, 105, 261-291.

93. Glass, A.M.W.; Macintyre, A.; Point, F. Free abelian lattice-ordered groups. Ann. Pure Appl. Log. 2005, 134, 265-283.

94. Alimov, N.G. On ordered semigroups. Izv. Akad. Nauk SSSR Ser. Math. 1950, 14, 569-576. (In Russian)

95. Hion, Y.V. Ordered semigroups. Izv. Akad. Nauk. SSSR Ser. Math. 1957, 21, 209-222. (In Russian)

96. Holman, E.W. Strong and weak extensive measurement. J. Math. Psychol. 1969, 6, 286-293.

97. De Miguel, J.R.; Candeal, J.C. Induráin. Archimedeaness and additive utility on totally ordered semigroups. Semigroup Forum 1996, 52, 335-347.

98. Candeal, J.C.; De Miguel, J.R.; Induráin, E. Extensive measurement: Continuous additive utility functions on semigroups. J. Math. Psychol. 1996, 40, 281-286.

99. Candeal, J.C.; De Miguel, J.R.; Induráin, E. Topological additively representable semigroups. J. Math. Anal. Appl. 1997, 210, 375-389.

100. Candeal, J.C.; De Miguel, J.R.; Induráin, E. Universal semigroups in additive utility. Semigroup Forum 1998, $56,288-296$. 
101. Candeal, J.C.; Induráin, E.; Olóriz, E. Weak extensive measurement without translation-invariance axioms. J. Math. Psychol. 1998, 42, 48-62.

102. Candeal, J.C.; Induráin, E.; Olóriz, E. Existence of additive utility on positive semigroups: An elementary proof. Ann. Oper. Res. 1998, 80, 269-279.

103. Candeal, J.C.; De Miguel, J.R.; Induráin, E. Existence of additive and continuous utility functions on ordered semigroups. J. Econ. 1999, 8, 53-68.

104. Candeal, J.C.; De Miguel, J.R.; Induráin, E. Expected utility from additive utility on semigroups. Theory Decis. 2002, 53, 87-94.

105. Fuchs, L. Partially Ordered Algebraic Systems; Pergamon Press: Oxford, UK, 1963.

106. Birkhoff, G. Lattice Theory, 3rd ed.; American Mathematical Society: Providence, RI, USA, 1967.

107. Davey, B.A.; Priestley, H.A. Introduction to Lattices and Order; Cambridge University Press: Cambridge, UK, 1990.

108. Neuefeind, W.; Trockel, W. Continuous linear representability of binary relations. Econom. Theory 1995, 6, 351-356.

109. Candeal-Haro, J.C.; Induráin-Eraso, E. A note on linear utility. Econom. Theory 1995, 6, 519-522.

110. Candeal, J.C.; Induráin, E.; Mehta, G.B. Order-preserving functions on ordered topological vector spaces. Bull. Aust. Math. Soc. 1999, 60, 55-65.

111. Bosi, G.; Campión, M.J.; Candeal, J.C.; Induráin, E.; Zuanon, M.E. Isotonies on ordered cones through the concept of a decreasing scale. Math. Soc. Sci. 2007, 54, 115-127.

112. Candeal, J.C.; Induráin, E.; Molina, J.A. Numerical representability of ordered topological spaces with compatible algebraic structure. Order 2012, 29, 131-146.

113. Berg, K.R. Independence and additive entropy. Proc. Am. Math. Soc. 1975, 51, 366-370.

114. Zanchini, Z.; Beretta, G.P. Recent progress in the definition of thermodynamic entropy. Entropy 2014, 16, $1547-1570$

115. Fishburn, P.C. Interval representations for interval orders and semiorders. J. Math. Psychol. 1973, 10, 91-105.

116. Doignon, J.P.; Ducamp, A.; Falmagne, J.C. On realizable biorders and the biorder dimension of a relation. J. Math. Psychol. 1884, 28, 73-109.

117. Candeal, J.C.; Induráin, E. Semiorders and thresholds of utility discrimination: Solving the Scott-Suppes representability problem. J. Math. Psychol. 2010, 54, 485-490.

118. Candeal, J.C.; Induráin, E.; Zudaire, M. Numerical representability of semiorders. Math. Soc. Sci. 2002, 43, 61-77.

119. Candeal, J.C.; Estevan, A.; Gutiérrez García, J.; Induráin, E. Semiorders with separability properties. J. Math. Psychol. 2012, 56, 444-451.

120. Estevan, A.; Gutiérrez García, J.; Induráin, E. Numerical representation of semiorders. Order 2013, 30, $455-462$.

121. S̀wistak, P. Some representation problems for semiorders. J. Math. Psychol. 1980, 21, 124-135.

122. Manders, K.L. On JND representations of semiorders. J. Math. Psychol. 1981, 24, 224-248.

123. Gensemer, S.H. On numerical representations of semiorders. Math. Soc. Sci. 1988, 15, 277-286.

124. Beja, A.; Gilboa, I. Numerical representations of imperfectly ordered preferences. A unified geometric exposition. J. Math. Psychol. 1992, 36, 426-449.

125. Pirlot, M.; Vincke, P. Semiorders: Properties, Representations, Applications; Kluwer: Dordrecht, The Netherlands, 1997.

126. Abrísqueta, F.J.; Candeal, J.C.; Induráin, E.; Zudaire, M. Scott-Suppes representability of semiorders: Internal conditions. Math. Soc. Sci. 2009, 57, 245-261.

127. Estevan, A. Generalized Debreu's open gap lemma and continuous representability of biorders. Order 2016, 33, 213-229.

128. Estevan, A.; Gutiérrez García, J.; Induráin, E. Further results on the continuous representability of semiorders. Int. J. Uncertain. Fuzziness Knowl. Based Syst. 2013, 21, 675-694.

129. Bosi, G.; Estevan, A.; Gutiérrez García, J.; Induráin, E. Continuous representability of interval orders: The topological compatibility setting. Int. J. Uncertain. Fuzziness Knowl. Based Syst. 2015, 23, 345-365.

130. Tversky, A. Intransitivity of preferences. Psychol. Rev. 1969, 76, 31-48.

131. Field, R.J. Limit cycle oscillations in the reversible oregonator. J. Chem. Phys. 1975, 63, 2289.

132. Cantor, G. Beiträge zur Begründung der transfinite Mengenlehre (I). Math. Ann. 1895, 46, 481-512. 
133. Cantor, G. Beiträge zur Begründung der transfinite Mengenlehre (II). Math. Ann. 1897, 49, 207-246.

134. Aleskerov, F.; Bouyssou, D.; Monjardet, B. Utility Maximization, Choice and Preference, 2nd ed.; Springer: Berlin/Heidelberg, Germany, 2007.

135. Skala, H.J. Nonstandard utilities and the foundations of game theory. Int. J. Game Theory 1974, 3, 67-81.

136. Campión, M.J.; Candeal, J.C.; Induráin, E. Representability of binary relations through fuzzy numbers. Fuzzy Sets Syst. 2006, 157, 1-19.

137. Candeal, J.C.; Gutiérrez García, J.; Induráin, E. Universal codomains to represent interval orders. Int. J. Uncertain. Fuzziness Knowl. Based Syst. 2009, 17, 197-219.

138. Bosi, G.; Gutiérrez García, J.; Induráin, E. Universal representability of total preorders and interval orders through a single function: The lattice approach. Order 2009, 26, 255-275.

139. Chipman, J.S. The foundations of utility. Econometrica 1960, 28, 193-224.

140. Giarlotta, A.; Watson, S. Pointwise Debreu lexicographic powers. Order 2009, 26, 377-408.

141. Bosi, G.; Isler, R. Representing preferences with nontransitive indifference by a single real-valued function. J. Math. Econom. 1995, 24, 621-631.

142. Skala, H.J. Non-Archimedean Utility Theory; Springer Science: Dordrecht, The Netherlands, 1975.

143. Schmaltz, M. Pierpaoli, E. (Eds.) Searching For New Physics At Small And Large Scales (TASI 2012); World Scientific: Singapore, 2012.

144. Steinhauser, M.O. Computational Multiscale Modeling of Fluids and Solids-Theory and Applications; Springer: Berlin/Heidelberg, Germany, 2010.

145. Nielsen, M.A. Conditions for a class of entanglement transformations. Phys. Rev. Lett. 1999, 83, 436.

146. Gielerak, R.; Sawerwain, M. Sorting of Quantum States with Respect to Amount of Entanglement Included. Commun. Comput. Inf. Sci. 2009 39, 11-18.

147. Cerf, N.J.; Adami, C. Negative entropy and information in quantum mechanics. Phys. Rev. Lett. 1997, 79, 5194-5197.

148. Campisi, M. Increase of Boltzmann entropy in a quantum forced harmonic oscillator. Phys. Rev. E 2008, $78,051123$.

149. Abe, S.; Okuyama, S. Similarity between quantum mechanics and thermodynamics: Entropy, temperature, and Carnot cycle. Phys. Rev. E 2011, 83, 021121.

150. Brandão, F.; Horodecki, M.; Ng, N.; Oppenheim, J.; Wehner, S. The second laws of quantum thermodynamics. Proc. Natl. Acad. Sci. USA 2015, 112, 3275-3279.

(C) 2018 by the authors. Licensee MDPI, Basel, Switzerland. This article is an open access article distributed under the terms and conditions of the Creative Commons Attribution (CC BY) license (http:/ / creativecommons.org/licenses/by/4.0/). 